\title{
Negative Regulation of Dopamine Transporter Endocytosis by Membrane-Proximal N-Terminal Residues
}

\author{
Tatiana Sorkina, Toni L. Richards, Anjali Rao, Nancy R. Zahniser, and Alexander Sorkin \\ Department of Pharmacology and Neuroscience Program, Anschutz Medical Center, University of Colorado Denver, Aurora, Colorado 80045
}

\begin{abstract}
The plasma membrane dopamine transporter (DAT) takes extracellular dopamine back up into dopaminergic neurons. Although the number of DATs at the cell surface is regulated by endocytosis and recycling, the molecular mechanisms that control this endocytic trafficking of DAT are not defined. To map the sequence motifs that are involved in constitutive DAT endocytosis, mutagenesis of human DAT tagged with yellow fluorescent protein (YFP) and an extracellular HA epitope was performed. Removal of the entire $\mathrm{N}$ terminus of DAT resulted in accumulation of the resulting DAT mutant (YFP-HA- $\Delta$ N-DAT) in early and recycling endosomes in HeLa and PAE cells, and in primary rat mesencephalic-striatal neuronal cocultures. This endosomal accumulation was due to rapid constitutive internalization of YFP-HA- $\Delta$ N-DAT by the clathrin-dependent pathway. Small deletions and multialanine substitutions in the $\mathrm{N}$ terminus revealed two molecular determinants within the membrane proximal residues 60 - 65 that are important for preventing rapid internalization of DAT. First, mutations of Arg60 or Trp63, leading to disruption of the "outward facing" DAT conformation, correlated with an increased pool of mobile DATs in the plasma membrane and accelerated constitutive internalization of the DAT mutants. Second, mutation of Lys 65 also correlated with elevated endocytosis. While none of these mutations alone recapitulated the marked endocytic phenotype of YFPHA- $\Delta$ N-DAT, simultaneous elimination of both the outward conformation of DAT and Lys65 resulted in DAT mutants that were rapidly internalized. Thus, our studies reveal a new link between DAT endocytosis and conformation-dependent uptake activity that represents a novel mode for regulating DAT function.
\end{abstract}

Key words: dopamine transporter; endocytosis; recycling; dopaminergic neurons; amphetamine; clathrin; fluorescence microscopy

\section{Introduction}

The plasma membrane dopamine transporter (DAT) is expressed exclusively in dopaminergic neurons in the CNS and functions to transport extracellular dopamine (DA) back into these neurons, thus providing temporal and spatial regulation of DA neurotransmission. DAT is a target of psychostimulants, such as amphetamines (AMPHs) and cocaine, and can be involved in the development of neurodegenerative diseases. DAT belongs to the SLC6 gene family of $\mathrm{Na}^{+} / \mathrm{Cl}^{-}$-dependent neurotransmitter transporters (Gether et al., 2006). These transporters have 12 transmembrane-spanning domains, intracellular $\mathrm{N}$ - and C-terminal tails, and a large extracellular loop 2.

The number of DAT molecules at the surface of neurons determines the efficiency of DA clearance in the brain. The surface levels of DAT depend on the rates of anterograde transport of the newly synthesized DAT from the endoplasmic reticulum to the plasma membrane and are also acutely regulated by endocytic trafficking. Recent studies demonstrated that endocytosis and recycling of DAT can be modulated by various intracellular sig-

\footnotetext{
Received July 11, 2008; revised Dec. 21, 2008; accepted Dec. 26, 2008.

This work was supported by National Institutes of Health Grants R01 DA014204, T32 AA007464, and K05 DA015050. We thank Kalen Dionne and Dr. David Price for help with neuronal cultures.

Correspondence should be addressed to Alexander Sorkin, Department of Pharmacology, Anschutz Medical Center, University of Colorado Denver, Mail Stop 8303, P.0. Box 6511, Aurora, C0 80045. E-mail: Alexander.sorkin@uchsc.edu.

DOI:10.1523/JNEUROSCI.3250-08.2009

Copyright $\odot 2009$ Society for Neuroscience $\quad$ 0270-6474/09/291361-14\$15.00/0
}

naling cascades as well as by DAT substrates and inhibitors, such as AMPHs and cocaine, respectively (for review, see Melikian, 2004; Zahniser and Sorkin, 2004; Gether et al., 2006; Fleckenstein et al., 2007).

Endocytic trafficking of transmembrane proteins is a highly dynamic process that is tightly regulated by the complex machinery of proteins associated with clathrin-coated pits and endosomes. However, the specific molecular mechanisms involved in DAT endocytosis and recycling are not well understood. Most experiments have been performed in model heterologous expression cell systems, rather than in dopaminergic neurons, because of technical difficulties in monitoring DAT trafficking in these neurons. Furthermore, many studies have focused on the effects of protein kinase C (PKC) on DAT endocytosis (Daniels and Amara, 1999; Melikian and Buckley, 1999; Sorkina et al., 2005, 2006; Miranda et al., 2007). Much less is known about basal, constitutive endocytosis of DAT although such endocytosis has been demonstrated in heterologous expression systems and dopaminergic neurons (Loder and Melikian, 2003; Sorkina et al., 2005, 2006). In non-neuronal cells, it has been shown that both constitutive and PKC-dependent endocytosis of DAT requires clathrin; however, this has not been confirmed in dopaminergic neurons. Ubiquitination of DAT was shown to be required for the PKC-dependent internalization of the transporter (Sorkina et al., 2006; Miranda et al., 2007). The amino acid residues 589-598 in the C-terminal tail of DAT have been proposed to be necessary for the constitutive internalization of DAT (Holton et al., 2005). 
However, the molecular mechanism by which these residues can mediate endocytosis or, indeed, any other molecular mechanisms mediating constitutive endocytosis are unknown.

The present studies were prompted by our unexpected observation of accumulation of a DAT mutant lacking the $\mathrm{N}$ terminus in endosomes. We then hypothesized that constitutive endocytosis of the full-length DAT is inhibited by the N-terminal domain. Using systematic mutagenesis of the $\mathrm{N}$ terminus, we found that residues 60-65, which are proximal to the first transmembrane domain of DAT, are critical for the retention of DAT at the plasma membrane and/or prevention of rapid constitutive endocytosis of DAT.

\section{Materials and Methods}

Reagents. Antibodies were purchased from the following sources: monoclonal rat antibody against the $\mathrm{N}$ terminus of DAT and rabbit polyclonal antibody to tyrosine hydroxylase (TH) from Millipore; mouse monoclonal antibody HA.11 from Covance; donkey anti-mouse antibodies conjugated with $\mathrm{Cy} 5$ or $\mathrm{Cy} 3$ from Jackson ImmunoResearch Laboratories; mouse monoclonal antibody to HA epitope (12CA5) were from Roche Applied Science; EEA.1 were from BD Transduction Laboratories; goat anti-rabbit antibody conjugated with Alexa-350 from Invitrogen; rabbit polyclonal antibody to clathrin heavy chain $(\mathrm{CHC})$ from Abcam Limited. PMA was purchased from Sigma. Transferrin conjugated with Texas Red (Tfn-TxR) and LysoTrackerRed were from Invitrogen. AMPH (Damphetamine sulfate salt) was from Sigma. Other chemicals were from Fisher Scientific.

Plasmids. The plasmid YFP-HA-DAT was described previously (Sorkina et al., 2006). An HA epitope tag was incorporated into YFP- $\Delta \mathrm{N}$ DAT construct (Sorkina et al., 2005) by site-directed mutagenesis as described previously (Sorkina et al., 2006) to generate YFP-HA- $\Delta \mathrm{N}$ DAT. YFP-HA- $\Delta 36-D A T$ was prepared by inserting a PCR fragment corresponding to residues 37-620 of human DAT into KpnI and SmaI restriction sites of pEYFP-C1 and incorporating an HA epitope by sitedirected mutagenesis as described previously (Sorkina et al., 2006). FlagHis-HA- $\Delta$ N-DAT was prepared by replacing the full-length human DAT cDNA in Flag-His-HA-DAT construct described previously (Sorkina et al., 2006) by the PCR fragment corresponding to residues $66-620$ obtained from YFP-HA- $\triangle \mathrm{N}-\mathrm{DAT}$. All alanine substitution mutants in the full-length YFP-HA-DAT construct were made using a Stratagene Quick-change mutagenesis kit according to the manufacture's protocol using YFP-HA-DAT as a template. The mutations were verified by automatic dideoxynucleotide sequencing.

Cell culture and transfections. Human HeLa cells were grown in DMEM containing 10\% fetal bovine serum (FBS, Hyclone). Porcine aortic endothelial (PAE) cells were grown in Ham's F12 medium containing 10\% FBS.

Primary neuronal cocultures of mesencephalon and striatum (MESSTR cocultures) were obtained from E15 Sprague Dawley rat embryos as described previously (Sorkina et al., 2006). Pregnant dams were purchased from Sasco Charles River. All animal use procedures were in accordance with the National Institutes of Health Guide for the Care and Use of Laboratory Animals and were approved by the Institutional Animal Care and Use committee at the University of Colorado Health Sciences Center. A mixture of mesencephalic cells $(480,000$ viable cells/ $\left.\mathrm{cm}^{2}\right)$ and striatal cells $\left(120,000 \mathrm{cells} / \mathrm{cm}^{2}\right)$ were plated into 24 -well dishes containing $12 \mathrm{~mm}$ glass coverslips pretreated with polyethylenimine and into $1 \mathrm{ml}$ of media. One milliliter of fresh media was added to the existing media $1 \mathrm{~d}$ after plating. Half of the media was replaced with fresh media every 2-3 d thereafter. The cultures were transfected in vitro on day 3-6.

An Effectene method (Qiagen) was used for DNA transfection of HeLa and PAE cells and Lipofectamine 2000 (Invitrogen) was used for MESSTR cocultures. HeLa and PAE cells stably expressing YFP-HA-DAT were selected by growing them in the presence of G418. siRNA transfections were performed as described previously (Sorkina et al., 2005). Control nontargeting siRNA (Dharmacon) was used in mock-transfections.

Single-cell internalization assay. This assay was performed as described previously (Sorkina et al., 2006). Briefly, the cells grown on glass coverslips were incubated with $1 \mu \mathrm{g} / \mathrm{ml}$ HA11 in the conditioned medium for
$60 \mathrm{~min}$ at $37^{\circ} \mathrm{C}$, in $5 \% \mathrm{CO}_{2}$ atmosphere and then incubated at $37^{\circ} \mathrm{C}$ in the same medium with vehicle or monensin $(25 \mu \mathrm{M})$ for $30 \mathrm{~min}$. The exception was the experiments with the AMPH treatment where the cells were incubated with HA11 and AMPH in Krebs-Ringer HEPES buffer (KRH; $120 \mathrm{~mm} \mathrm{NaCl}, 4.7 \mathrm{~mm} \mathrm{KCl}, 2.2 \mathrm{~mm} \mathrm{CaCl}_{2}, 1.2 \mathrm{~mm} \mathrm{Mg} \mathrm{SO}, 1.2 \mathrm{~mm}$ $\mathrm{KH}_{2} \mathrm{PO}_{4}, 10$ mm glucose, 10 mm HEPES, pH 7.4). In CHC siRNA knockdown experiments, the cells were incubated with $\mathrm{HA} 11$ at $20^{\circ} \mathrm{C}$, then washed and further incubated without $\mathrm{HA} 11$ at $37^{\circ} \mathrm{C}$ for $30 \mathrm{~min}$. In all cases, after $37^{\circ} \mathrm{C}$ incubation, the cells were then washed 3 times in icecold F12 medium, then once with ice-cold $\mathrm{Ca}^{2+}, \mathrm{Mg}^{2+}$-free PBS (CMFPBS) and fixed with freshly prepared $4 \%$ paraformaldehyde for $15 \mathrm{~min}$ at room temperature. The cells were stained with saturating amounts of secondary anti-mouse antibody conjugated with saturating amounts of Cy5 by incubating in CMF-PBS containing 0.5\% BSA at room temperature for 60 min to occupy surface HA11. After washing, the cells were first permeabilized by a 10 -min incubation in CMF-PBS containing $0.1 \%$ saponin $/ 0.5 \%$ BSA at room temperature and then incubated with the same secondary antibodies conjugated with $\mathrm{Cy} 3$ in the same saponincontaining buffer for $45 \mathrm{~min}$ to label internalized HA11. Both primary and secondary antibody solutions were precleared by centrifugation at $100,000 \times g$ for $20 \mathrm{~min}$. After staining, the coverslips were mounted in Mowiol (Calbiochem).

Fluorescence microscopy. To obtain high resolution three-dimensional images of the cells, a $Z$-stack of $x-y$ images were acquired through Cy5, $\mathrm{Cy} 3$ (for $\mathrm{Cy} 3$ or TexasRed fluorescence) and FITC (YFP fluorescence) filter channels using a Marianas Imaging workstation and SlideBook 4.2 software (Intelligent Imaging Innovation) as described previously (Sorkina et al., 2005). Neuronal cultures were additionally stained with rabbit antibody to TH followed by secondary antibody conjugated with Alexa-350 (imaged through aminomethylcoumarin acetate or DAPI filter channels). In CHC siRNA experiments, the cells were additionally stained with rabbit $\mathrm{CHC}$ antibody followed by secondary Alexa-350 conjugated antibody. Typically, 34 or 12 serial two-dimensional images were recorded at 200 or $400 \mathrm{~nm}$ intervals, respectively. All image acquisition settings were identical in each experiment. The $Z$-stack of images obtained was deconvoluted using a modification of the constrained iteration method (Gaussian noise smoothing) (34-stack) or a nearest neighbor algorithm (12-stack).

In endocytosis assays, quantification of the relative amount of Cy5 and Cy3 fluorescence in the cell was performed using the statistics module of SlideBook4.2 software. The background-subtracted three-dimensional (3D) images were segmented using a minimal intensity of YFP as a low threshold. The integrated voxel intensity of Cy5 and Cy3 in each cell in YFP-containing voxels was then quantitated, and the ratio of total Cy3 to $\mathrm{Cy} 5$ signals was calculated. The background Cy3/Cy5 ratio was measured in several regions of cells not containing endosomes and subtracted from the raw $\mathrm{Cy} 3 / \mathrm{Cy} 5$ ratio to obtain a specific ratio of internalized to surface HA11 (Cy3/Cy5) that was used to estimate the rates of endocytosis of HA11:YFP-HA-DAT complexes. The absolute values of Cy3/Cy5 ratio measured for the same cell clones displayed some variability in different experiments due to the use of different batches of secondary Cy3- and Cy5-conjugated antibodies.

Fluorescence recovery after photobleaching. The cells expressing YFPHA-DAT were grown on coverslips and mounted in a microscope chamber for live-cell imaging. Fluorescence recovery after photobleaching (FRAP) experiments were performed using a Marianas Imaging workstation equipped with a micropoint FRAP system (Photonic Instruments). Images were acquired before and after photobleaching of YFP using a dye laser at $515 \mathrm{~nm}$, a $200 \mathrm{~ms}$ exposure time, and $2 \mathrm{~s}$ intervals between images. In these experiments, the excitation intensity during image acquisition was reduced due to the introduction of a 50/50 dichromatic for the FRAP system to achieve simultaneous photobleaching and image acquisition. Because filopodia and ruffles are highly dynamic at $37^{\circ} \mathrm{C}$, our measurements were performed at room temperature $\left(22^{\circ} \mathrm{C}\right)$. After the first image, an area of the cell (diameter $\sim 3 \mu \mathrm{m}$ ) was rapidly irradiated by the pulse laser at $515 \mathrm{~nm}$, and the time-lapse imaging was immediately continued for $78 \mathrm{~s}$. Reduction of the initial YFP fluorescence intensity down to a level of $0-5 \%$ of the intensity of the same region before photobleaching was observed after two rounds of photobleaching (total time $<1 \mathrm{~s}$ ). The mean fluorescence intensity of YFP in the photo- 
bleached area (ROI-1), before and after photobleaching, was measured. To control for cell movement and fluorescence fluctuations independent of bleaching, the mean fluorescence intensity of unbleached regions of the cell (ROI-2) was measured. The experiments, in which minimal changes in ROI-2 s occurred, were used for FRAP calculations. Mean fluorescence intensities in the ROI-1 and ROI-2 were measured under the same condition for each data set. All experiments were performed under identical microscope settings for the laser energy input and the fluorescence detection. Recovery curves were calculated using nonlinear regression analysis in GraphPad Prism 4 to determine the mobile fraction $\left(M_{\mathrm{f}}\right)$ and diffusion time $\left(\tau_{\mathrm{D}}\right)$ (Yguerabide et al., 1982). The diffusion coefficient $D_{\mathrm{t}}$ was determined using the two-dimensional diffusion equation as described previously (Axelrod et al., 1976).

Immunoprecipitation. The cells grown in 12-well dishes were incubated with HA11 for 90 min at $37^{\circ} \mathrm{C}$, placed on ice and washed 5 times with F12 medium followed by rinsing with CMF-PBS. The proteins were solubilized in Triton X-100/deoxycholate/glycerol/HEPES lysis buffer with protease and phosphatase inhibitors for $30 \mathrm{~min}$ at $4^{\circ} \mathrm{C}$ (Sorkina et al., 2005). The lysate was then centrifuged at $16,000 \times g$ for $10 \mathrm{~min}$ to remove insoluble material. Lysates were precipitated with Protein G-Sepharose. Immunoprecipitates were resolved by electrophoresis and probed with HA11 or DAT antibodies, followed by chemiluminescence detection as described previously (Sorkina et al., 2005).

Kinetic analysis of DA uptake by HA-DAT in PAE cells. The cells were grown in 24-well plates for $2 \mathrm{~d}$. The cells were rinsed at $4^{\circ} \mathrm{C}$ and then assayed in $\mathrm{KRH}$ supplemented with $10 \mu \mathrm{M}$ pargyline, $10 \mu \mathrm{M}$ ascorbic acid, and $10 \mu \mathrm{M}$ catechol. Assays $(0.5 \mathrm{ml})$ included $50 \mathrm{~nm}\left[{ }^{3} \mathrm{H}\right] \mathrm{DA}$ (Perkin-Elmer Life Sciences) and increasing concentrations of unlabeled DA $(0,0.05,0.5,1,5,10$, and $50 \mu \mathrm{M})$. Nonspecific [ $\left.{ }^{3} \mathrm{H}\right] \mathrm{DA}$ accumulation was determined in the presence of $1 \mathrm{~mm}(-)$ cocaine $\mathrm{HCl}$ (National Institute on Drug Abuse/RTI International). After $10 \mathrm{~min}$ of incubation at $22^{\circ} \mathrm{C}$, uptake was terminated by quickly washing the cells three times with $0.5 \mathrm{ml}$ ice-cold $\mathrm{KRH}$. Cells were then solubilized in $0.5 \mathrm{ml} 5 \%$ trichloroacetic acid for $60 \mathrm{~min}$ with gentle shaking. Accumulated $\left[{ }^{3} \mathrm{H}\right] \mathrm{DA}$ was determined by liquid scintillation counting. $K_{\mathrm{m}}$ and $V_{\max }$ values for $\left[{ }^{3} \mathrm{H}\right] \mathrm{DA}$ uptake were calculated with nonlinear regression fitting using GraphPad Prism software.

Internalization assay using ${ }^{125}$ I-labeled HA antibody. Fifty to sixty micrograms of 12CA5 antibody were iodinated using Iodobeads (Pierce). The PAE cells stably expressing the full-length or mutant YFP-HA-DAT were grown in 12-well dishes. The cells were preincubated in conditioned medium containing $20 \mu \mathrm{g} / \mathrm{ml}$ nonspecific mouse IgG at $37^{\circ} \mathrm{C}$ and then incubated with $1 \mu \mathrm{g} / \mathrm{ml}{ }^{125} \mathrm{I}$-HA for $60 \mathrm{~min}$ in the binding medium (F12, $0.1 \%$ BSA) supplemented with $20 \mu \mathrm{g} / \mathrm{ml}$ nonspecific mouse IgG at $37^{\circ} \mathrm{C}$, followed by addition of the vehicle (methanol) or monensin (final concentration of $25 \mu \mathrm{M}$ ) and further incubation for $30 \mathrm{~min}$ at $37^{\circ} \mathrm{C}$. The cells were then washed three times with cold F12 medium, incubated with cold acetic acid buffer containing $0.2 \mathrm{M}$ acetic acid, $0.5 \mathrm{M} \mathrm{NaCl}, \mathrm{pH} 2.8$, for $5 \mathrm{~min}$ and then additionally rinsed with the same solution. Both the 5 min incubation and the subsequent rinse were combined to determine the amount of the surface ${ }^{125} \mathrm{I}-\mathrm{HA}$. The cells were then solubilized in $1 \mathrm{~N}$ $\mathrm{NaOH}$ to determine the amount of internalized ${ }^{125} \mathrm{I}-\mathrm{HA}$. The backgrounds were determined by using parental PAE cells not expressing YFP-HA-DAT in parallel to cells expressing DAT, or by using an excess of the HA11 antibody. Both types of background measurements yielded essentially similar values. The endocytic rate was expressed as the ratio of the amounts of specific internalized and surface radioactivity.

Statistical analysis. The statistical significance of the endocytosis rates of various DAT mutants were analyzed by unpaired $t$ tests. Significant differences were defined as those with $p<0.05$.

\section{Results}

Endosomal accumulation of DAT lacking the N-terminal tail

To study the role of the N-terminal tail of DAT in its endocytic trafficking, the first 65 aa were replaced by YFP. Visual examination of HeLA and PAE cells transiently or stably expressing YFP$\Delta \mathrm{N}$-DAT revealed significant accumulation of YFP- $\Delta \mathrm{N}-\mathrm{DAT}$ in intracellular compartments under steady-state conditions of cell growth. To investigate the mechanisms underlying this abnormal subcellular distribution of DATs lacking the N-terminal tail, a $\Delta \mathrm{N}$-DAT mutant with an HA epitope inserted into the extracellular loop 2 (YFP-HA- $\Delta$ N-DAT) was generated and stably expressed in both HeLa and PAE cells. The HA antibody (HA11) uptake assay (Sorkina et al., 2006) was then used to compare DAT endocytosis in HeLa and PAE cells stably expressing the YFP-HA$\Delta \mathrm{N}$-DAT mutant or full-length YFP-HA-DAT (Fig. 1). In this assay, the cells were allowed to bind and internalize HA11 and fixed. Surface-exposed HA11:DAT complexes were then labeled with secondary Cy5-conjugated antibodies. Last, the cells were permeabilized, and internalized HA11:DAT complexes were labeled with secondary Cy3-conjugated antibodies.

The incubation of YFP-HA- $\Delta \mathrm{N}$-DAT-expressing cells with HA11 resulted in accumulation of HA11-DAT complexes in the intracellular compartments (Cy3 fluorescence) that were also positive for YFP but negative for Cy5 fluorescence, suggesting that these compartments are endosomes (Fig. 1). The endosomes containing YFP-HA- $\Delta$ N-DAT contained vesicular and tubular subcompartments, characteristic of the heterologous morphology of early and recycling endosomes. Not all YFP-containing intracellular compartments were also $\mathrm{Cy} 3$ positive. This result was expected because during the 90 min of HA11 uptake, HA11 would reach only a limited pool of endosomes containing YFPHA- $\Delta$ N-DAT, which would have been accumulated in endosomes over the long period of time before incubation of the cells with HA11. There was significantly less intracellular YFP and Cy3 fluorescence (internalized HA11-DAT complexes) in endosomes of cells expressing the control full-length YFP-HA-DAT (Fig. 1). The increased accumulation of HA11:YFP-HA- $\Delta$ N-DAT complexes in endosomes was observed in several single-cell clones, as well as in pools of stably transfected cells and in cells transiently transfected with YFP-HA- $\Delta$ NDAT. The endosomal concentration of HA11 was also observed in cells expressing $\Delta \mathrm{N}$-DAT tagged with small epitope tags (Flag and $10 \times$ His) instead of YFP (data not shown).

To examine endocytosis of YFP-HA- $\Delta$ N-DAT in neurons, YFP-HA- $\Delta$ N-DAT was transiently expressed in rat embryonic MES-STR neuronal cocultures. The neurons were incubated with $\mathrm{HA} 11$ at $37^{\circ} \mathrm{C}$ to allow binding of HA11 to all surface-exposed YFP-HA- $\Delta$ N-DAT molecules and endocytosis of the antibodyDAT complex. To compare the localization of YFP-HA- $\Delta N-$ DAT and endogenous rat DAT, the rat DAT was labeled with antibodies against the $\mathrm{N}$ terminus and $\mathrm{Cy} 3$-conjugated secondary antibody, whereas transfected YFP-HA- $\Delta$ N-DAT was detected by HA11 and Cy5-conjugated secondary antibody (surface plus internalized) and YFP (total: surface, internalized and newly sensitized in endoplasmic reticulum and Golgi) fluorescence. As shown in Figure 2A, HA11 was partially colocalized with endogenous DAT. The colocalization was especially evident at the edges of neuronal soma (presumably, plasma membrane) and long processes. There appeared to be an increased accumulation of YFP-HA- $\Delta$ N-DAT in the somatodendritic compartment of the DA neurons; however, quantitative analysis of the distribution of the transfected versus endogenous DATs was not possible due to the low efficiency of transfection of dopaminergic neurons and inability to distinguish surface and internalized endogenous transporters. It should be noted that colocalization could be due in part to dimerization of rat DAT and YFP-HA- $\Delta \mathrm{N}-\mathrm{DAT}$ (Sorkina et al., 2003).

To directly compare endocytosis of the full-length DAT and an amino-terminally truncated DAT mutant in neurons, MES-STR cocultures were transfected with YFP-HA-DAT or 


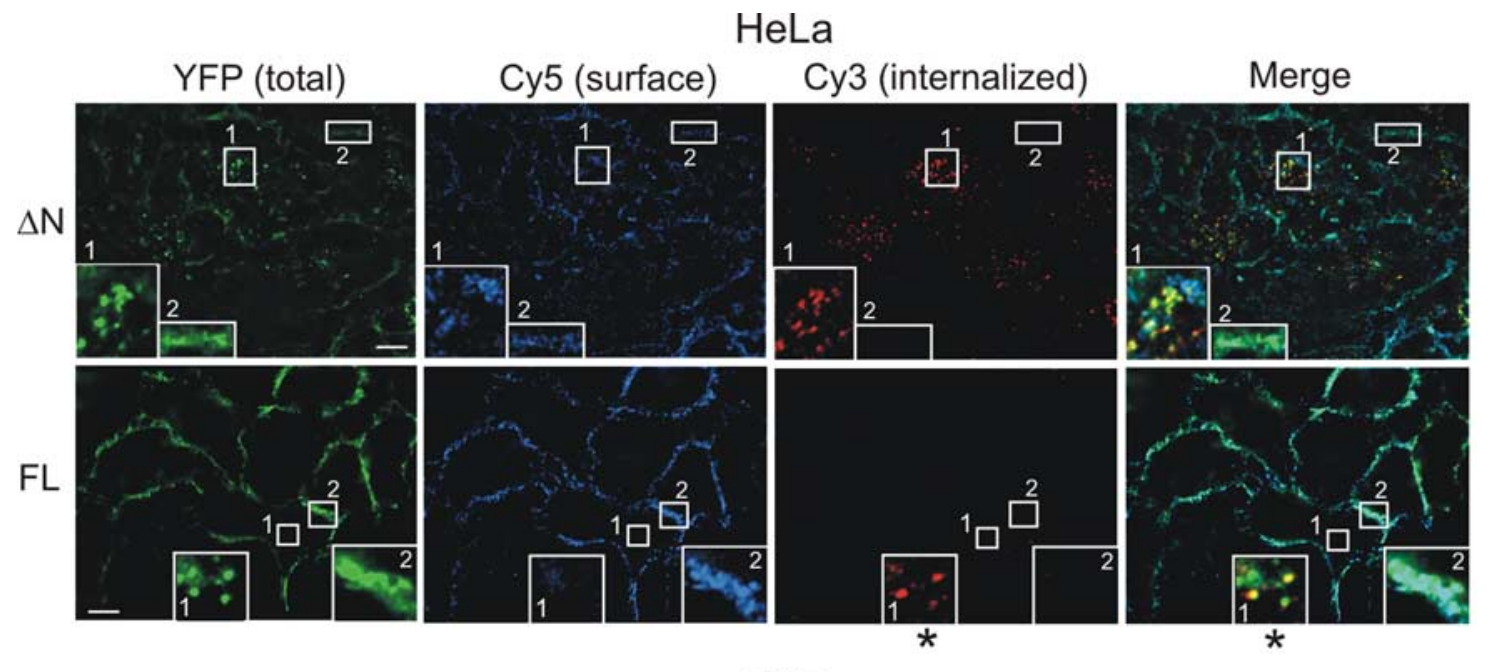

PAE
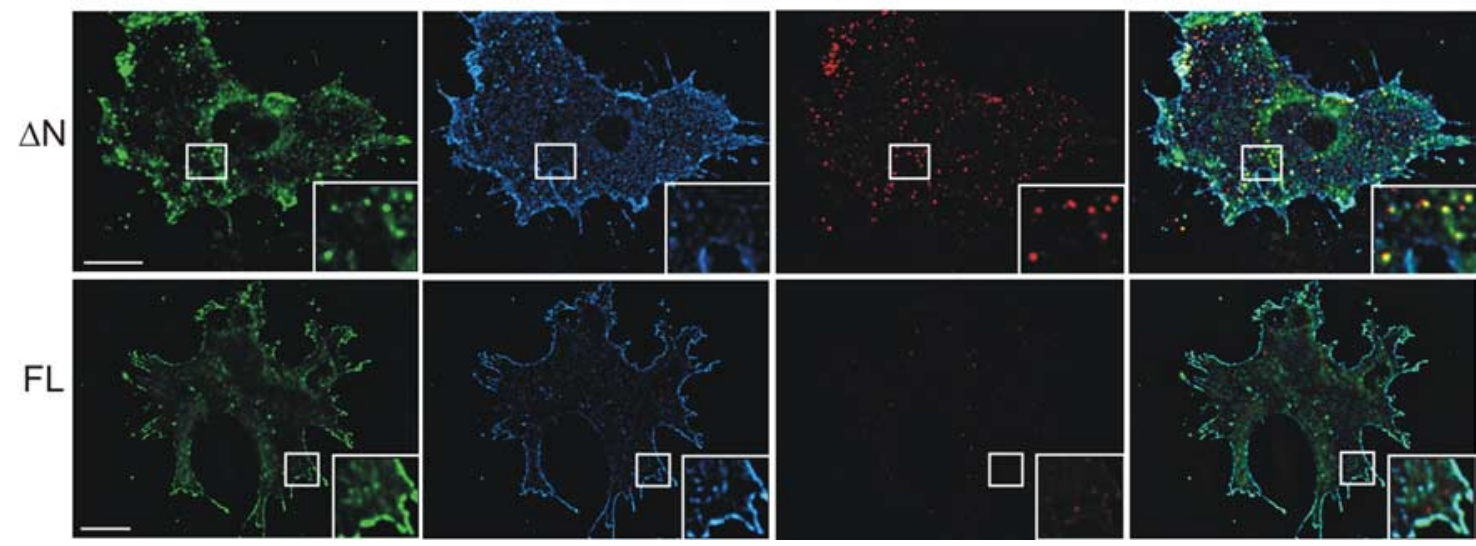

Figure 1. Accumulation of YFP-HA- $\Delta N-D A T$ in endosomes. HeLa $(\boldsymbol{A})$ or PAE $(\boldsymbol{B})$ cells stably expressing YFP-HA- $\Delta \mathrm{N}-\mathrm{DAT}(\Delta \mathrm{N})$ or YFP-HA-DAT (full-length; FL) were incubated with HA11 antibodies at $37^{\circ} \mathrm{C}$ for $90 \mathrm{~min}$ and fixed. Nonpermeabilized cells were stained with Cy5-tagged anti-mouse secondary lgG to detect surface HA11:YFP-HA-DAT complexes, followed by cell permeabilization and staining with Cy3-conjugated secondary antibodies to mark internalized HA11 as described in Materials and Methods. Merge images represent an overlap of YFP, Cy3, and Cy5 images. Optical sections through the middle of the cells are presented. The intensity scales of each fluorescence channel are identical in images of HA- $\triangle N$-DAT or YFP-HA-DAT. Insets represent high magnification images of the regions indicated by white rectangles. To enhance the appearance of Cy3-containing endosomes in HeLa cells expressing FL YFP-HA-DAT, the Cy3 intensity range in inset $\# 1$ (marked by asterisks) is set using a fivefold lesser maximal value of fluorescence intensity compared with other insets and full images of Cy3 fluorescence. Scale bars, $10 \mu \mathrm{m}$.

YFP-HA- $\Delta$ N-DAT. The HA11 endocytosis assay with subsequent detection of the surface and internalized pools of HA11DAT complexes was performed as described in Figure 1. Dopaminergic neurons were identified by staining with antibody to TH. It should be noted that immunofluorescence analysis of these cultures revealed that there was only a partial overlap of endogenous DAT and TH staining and $\sim 40 \%$ of stained neurons were positive for either DAT or TH (data not shown), suggesting the presence of a pool of immature dopaminergic neurons in these cultures. Therefore, a 3D stack of images was acquired from YFP-HA-tagged DAT-expressing neurons that were TH-positive and cells that did not contain a detectable amount of TH but displayed typical morphology of dopaminergic neurons (very long, thin processes with numerous varicosities). Figure $2 B$ illustrates representative observations of constitutive endocytosis of both full-length and truncated YFP-HA-DATs in distal thin (presumably, axonal) processes and somatodendritic compartments of neurons as revealed by the detection of vesicles stained with Cy3 and YFP, but not with Cy5. Quantification of the Cy3/Cy5 ratio, which reflects the endocytosis rate, revealed that there was greater endosomal accumulation of YFP-HA- $\Delta$ N-DAT, compared with YFP-
HA-DAT, in distal processes and somatic compartments (Fig. $2 C)$. Furthermore, there was no statistically significant difference in the endocytic rates of HA11 in TH-positive and THnegative neurons. The experimental results shown in Figure 2 demonstrated that the endocytic trafficking of YFP-HA- $\Delta \mathrm{N}-$ DAT in neurons resembles its trafficking in non-neuronal cells.

Increased constitutive internalization of YFP-HA- $\Delta N-D A T$ measured in the presence of the recycling inhibitor monensin The accumulation of YFP-HA- $\Delta$ N-DAT in endosomes could be due to either increased constitutive internalization or decreased recycling of this mutant, compared with the fulllength DAT. To minimize recycling of HA11:DAT from endosomes, the cells were treated with the proton ionophore monensin, an inhibitor of recycling from acidic endosomes (Sorkina et al., 2005). As expected, monensin caused accumulation of the full-length YFP-HA-DAT in endosomes of stably transfected PAE cells (colocalized YFP and HA11/Cy3 fluorescence), which can be attributed to constitutive endocytosis in the absence of recycling (Fig. 3). In cells expressing YFP-HA$\Delta \mathrm{N}$-DAT, monensin treatment resulted in even more dramatic endosomal accumulation of this mutant (Fig. 3). Very 


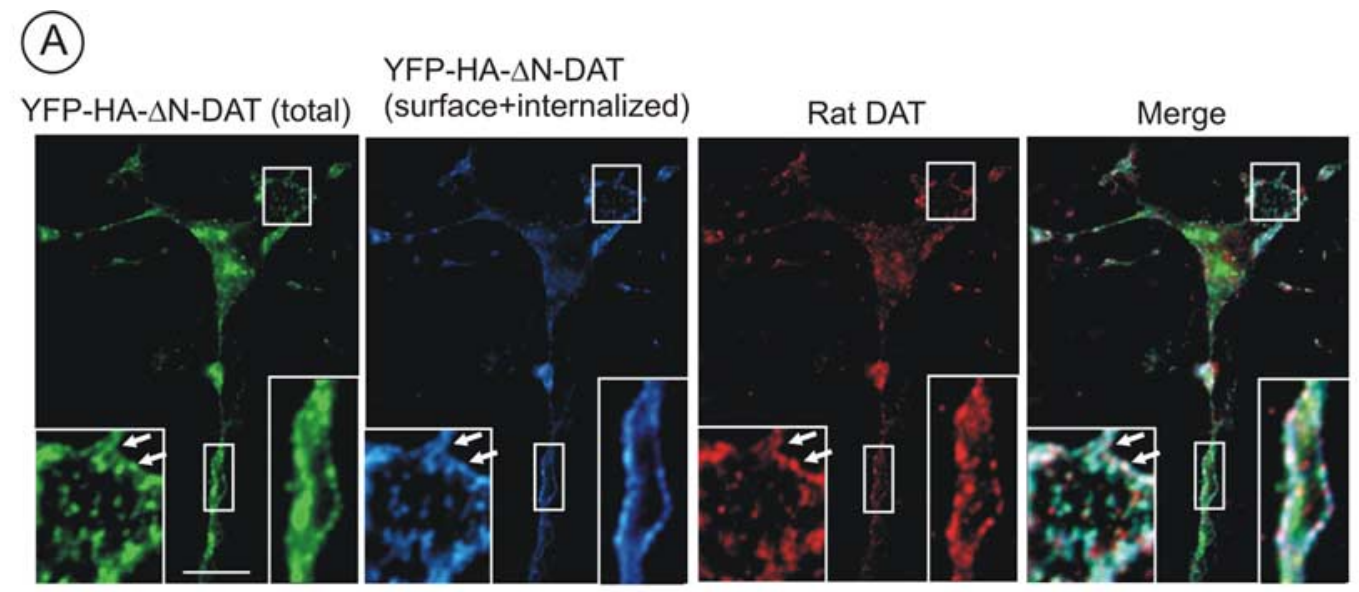

(B)
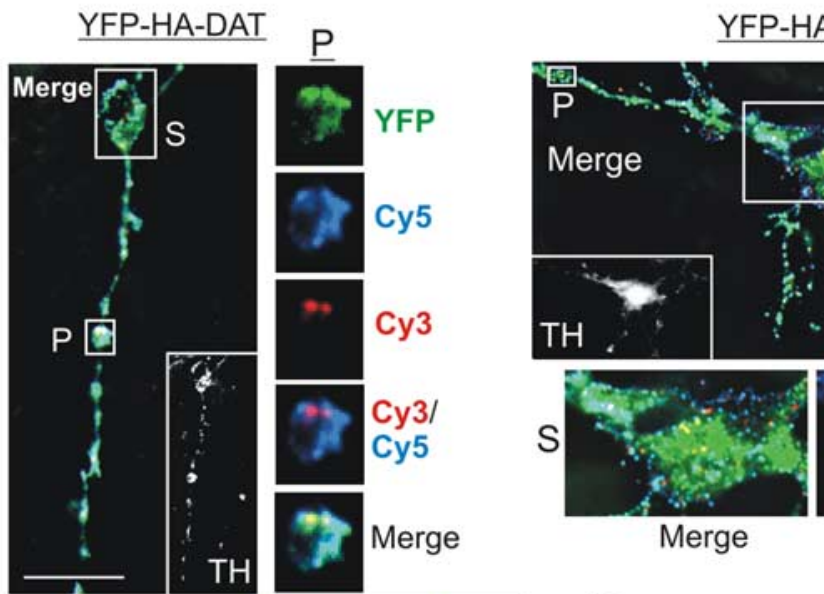

Merge

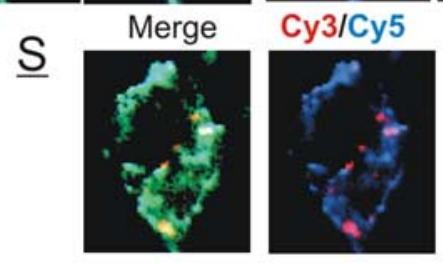

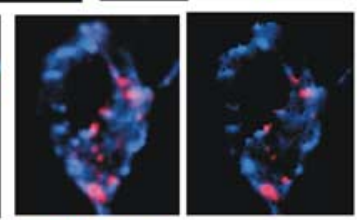

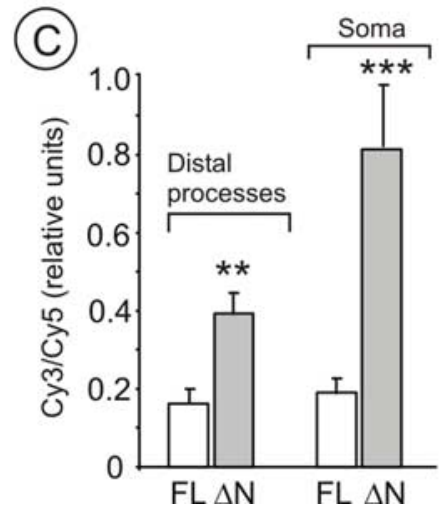

Figure 2. Localization of YFP-HA- $\triangle N$-DAT and endogenous DAT in rat MES-STR neuronal cocultures. A, YFP-HA- $\Delta N$-DAT was transiently expressed in MES-STR cocultures. After $3 \mathrm{~d}$, the neurons were incubated with $\mathrm{HA} 11$ at $18^{\circ} \mathrm{C}$ for $30 \mathrm{~min}$, then at $37^{\circ} \mathrm{C}$ for $30 \mathrm{~min}$ and fixed. The cells were then permeabilized and stained with Cy5-tagged anti-mouse secondary lgG to detect all surface and internalized HA11/YFP-HA- $\triangle N$-DAT complexes. Rat anti-DAT (N terminus specific), which does not recognize YFP-HA- $\triangle N$-DAT, was used to detect endogenous rat DAT (Cy3-labeled secondary anti-rat lgG). Merge images represent an overlap of YFP, Cy3, and Cy5 images. Optical sections through the middle of the neurons are presented. Insets represent high-magnification images of the regions indicated by white rectangles. Arrows indicate colocalization of endogenous rat DAT and YFP-HA- $\Delta \mathrm{N}$-DAT at the surface. Scale bars, $10 \mu \mathrm{m}$. B, YFP-HA-DAT and YFP-HA- $\Delta \mathrm{N}-\mathrm{DAT}$ were transiently expressed in MES-STR cocultures, and the neurons were incubated with HA11 as in Figure 1. Fixed, nonpermeabilized neurons were stained with Cy5-tagged anti-mouse secondary IgG to detect surface HA11:YFP-HA-DAT complexes, followed by cell permeabilization and staining with Cy3-conjugated secondary antibodies to mark internalized HA11 as described in Materials and Methods. After saponin permeabilization, the cells were simultaneously incubated with rabbit antibody to TH. Finally, the cells were incubated with secondary anti-rabbit IgG conjugated with Alexa-350. Insets at the right bottom corner of the main images represent an image of the TH staining. Individual optical sections are presented. Merge images represent an overlap of YFP, Cy3, and Cy5 images. Overlaps of Cy5 and Cy3 images (Cy3/Cy5) are shown to compare internalized and surface staining of HA11-DAT complexes. Insets represent high-magnification images of the regions of distal processes (P) and cell soma (S) indicated by white rectangles. Scale bars, $10 \mu \mathrm{m}$. C, Quantification of the endocytosis rates from the experiments exemplified in $\boldsymbol{B}$. The mean Cy3/Cy5 ratios of internalized/surface HA11 ( \pm SEM) were obtained from the values of Cy $3 / C y 5$ ratio calculated either for the entire length of each distal processes containing varicosities present in the image $(N=$ 20) or for the whole-cell soma $(N=9) .{ }^{* *} p<0.005 ;{ }^{* *} p<0.001$ (unpaired $t$ test).

weak surface staining of YFP-HA- $\Delta \mathrm{N}-\mathrm{DAT}$ was detected in the monensin-treated cells, indicative of a substantial downregulation of surface YFP-HA- $\Delta \mathrm{N}$-DATs under the condition of inhibited recycling. These data suggest that YFP-HA- $\Delta \mathrm{N}-$
DAT is efficiently recycled in the absence of monensin and that the accumulation of YFP-HA- $\Delta \mathrm{N}$-DAT in endosomes is primarily due to the rapid constitutive internalization, rather than slow recycling. 
YFP-HA- $\Delta$ N-DAT is constitutively internalized via the clathrin-dependent pathway into early and recycling endosomes

We have previously demonstrated that wild-type human DAT is constitutively internalized via clathrin-coated pits in HeLa and PAE cells (Sorkina et al., 2005). To examine whether rapid constitutive internalization of YFP-HA- $\Delta$ N-DAT is also clathrin-dependent, HeLa/YFP-HA- $\Delta \mathrm{N}$ DAT cells were transfected with $\mathrm{CHC}$ siRNA (Sorkina et al., 2005, 2006), and an HA11 endocytosis assay was carried out. Staining of siRNA-treated cells with CHC antibody revealed that $\mathrm{CHC}$ was depleted in $90-95 \%$ of cells. To directly assess the effect of CHC depletion on endocytosis, images were acquired from cells lacking coated pits together with neighboring cells that were not affected or partially affected by the CHC siRNA (Fig. 4A). As judged from the comparison of the amount of the punctuate Cy3 fluorescence (internalized HA11) in these two groups of cells, knock-down of CHC markedly reduced the uptake of HA11 into endosomes (Fig. 4A). These data suggest that the constitutive internalization of YFPHA- $\Delta N-D A T$ is clathrin-dependent. Essentially identical results were obtained in PAE cells stably expressing YFP-HA$\Delta$ N-DAT (data not shown).

To define the compartments where YFP-HA- $\Delta N-D A T$ is accumulated as the result of constitutive endocytosis, the PAE/YFP-HA- $\Delta$ N-DAT cells were continuously incubated with Tfn-TxR, which is internalized via the clathrin pathway into early and recycling endosomes. As seen in Figure 4B, YFP-HA$\Delta \mathrm{N}$-DAT was partially, but significantly, colocalized in Tfn-TxR-containing endosomes. Furthermore, YFP-HA- $\Delta$ NDAT was partially colocalized with EEA.1, a marker of early and intermediate endosomes (Fig. 4C). These data suggest that internalized YFP-HA- $\Delta \mathrm{N}-\mathrm{DAT}$ is located in early and recycling endo-

somes. There was much less colocalization of YFP-HA- $\Delta N-$ DAT with LysoTrackerRed, which primarily marks endosomes with an internal $\mathrm{pH}$ of $\leq 5.5$, such as a subset of multivesicular bodies, late endosomes and lysosomes (Fig. $4 D$ ). Interestingly, some large endosomes of complex shape containing LysoTracker were decorated by clusters of YFPHA- $\Delta$ N-DAT (Fig. $4 B$, high magnification insets). This pattern of localization indicates that a small pool of YFP-HA- $\Delta \mathrm{N}-$ DAT was located in small endosomes tethered to the multivesicular bodies or incorporated into the limiting membrane of multivesicular bodies, which is a subcompartment of these endosomes capable of cargo recycling. Altogether, the data in Figure 4 suggest that the constitutive endocytic pathway of YFP-HA- $\Delta$ N-DAT largely resembles that of the trans-
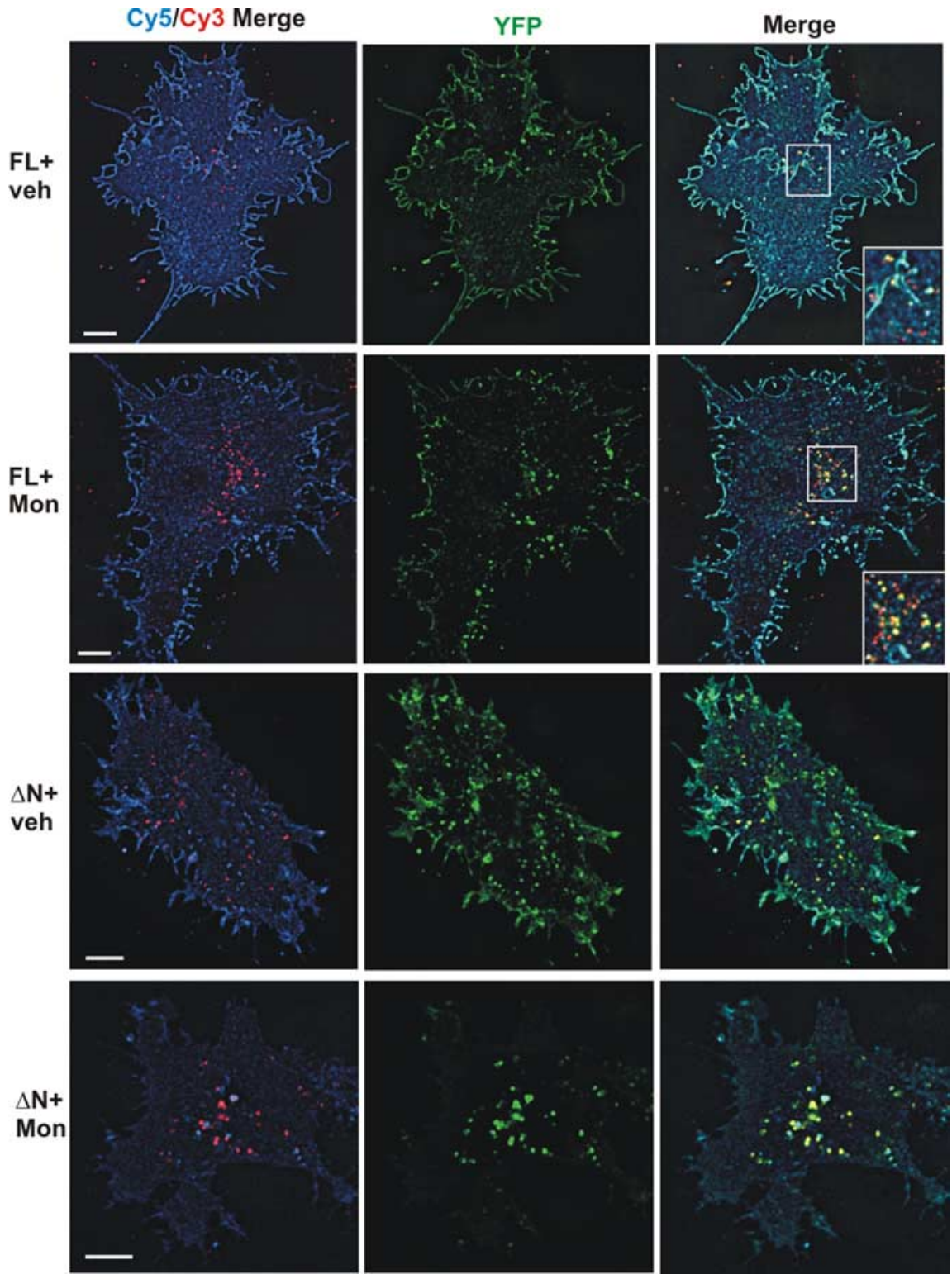

Figure 3. Accumulation of YFP-HA- $\triangle \mathrm{N}-\mathrm{DAT}$ in endosomes in cells treated with monensin. PAE cells stably expressing YFP-HA$\triangle \mathrm{N}$-DAT $(\triangle \mathrm{N})$ or YFP-HA-DAT (FL) were incubated with HA11 antibodies at $37^{\circ} \mathrm{C}$ for $60 \mathrm{~min}$, then with vehicle (veh) or monensin (25 $\mu \mathrm{m} ; \mathrm{Mon})$ at $37^{\circ} \mathrm{C}$ for $30 \mathrm{~min}$ and fixed. Staining with Cy5- (surface HA11) and Cy3-conjugated secondary antibodies (interimages. Overlap of Cy5 and Cy3 images (Cy3/Cy5 Merge) are shown to compare internalized and surface staining of HA11-DAT complexes. Insets represent high-magnification images of the endosome-rich regions of FL-expressing cells indicated by white rectangles. Optical sections through the middle of the cells are presented. Scale bars, $10 \mu \mathrm{m}$.

ferrin receptor, e.g., YFP-HA- $\Delta$ N-DAT is internalized via the clathrin-dependent mechanism and accumulates in early and recycling endosomal compartments.

The endocytosis-inhibitory signal is located in the juxtamembrane region of the $\mathrm{N}$ terminus of DAT

The data in Figures 1-4 demonstrated that removal of the $\mathrm{N}$ terminus of human DAT accelerates its constitutive endocytosis. This mutation could produce such an effect by relieving intramolecular and/or intermolecular interactions of DAT N-terminal residues that are responsible for inhibition of constitutive endocytosis. To define the molecular determinants in the N-terminal tail that mediate this "endocytosis-inhibitory" function, various amino acid residues in this part of DAT molecule were deleted or 


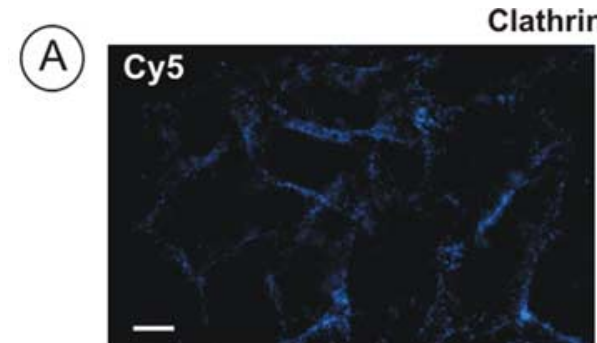

Clathrin siRNA


B
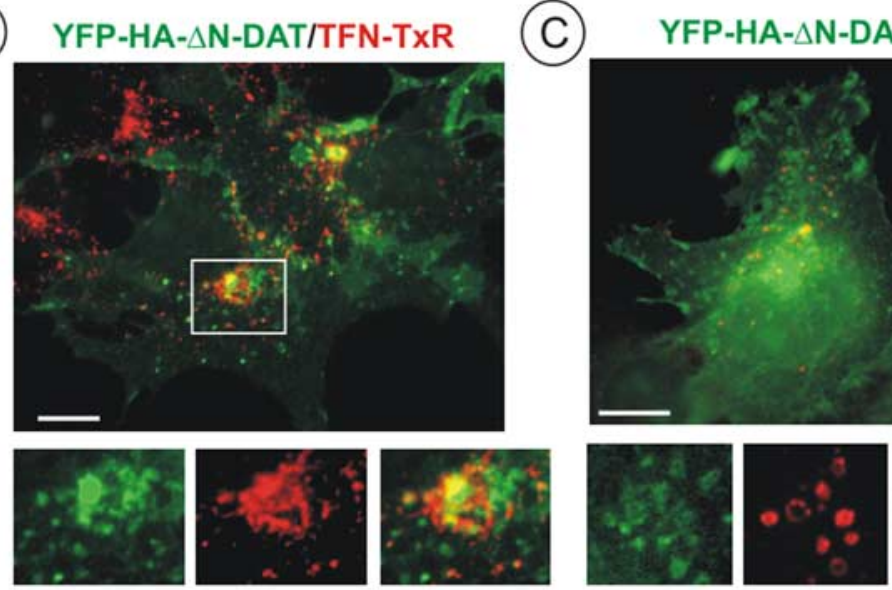

(D)

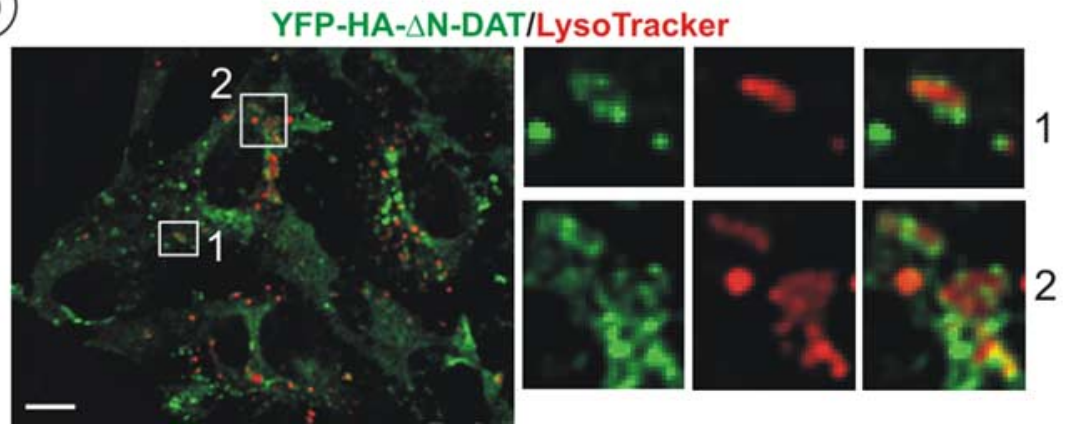

Figure 4. YFP- $\triangle N-H A-D A T$ is internalized via a clathrin-dependent pathway into early, recycling, and multivesicular endosomes. $\boldsymbol{A}$, HeLa/YFP- $\Delta \mathrm{N}$-HA-DAT cells were transfected with CHC siRNA. After $3 \mathrm{~d}$, HA11 endocytosis assays were performed as described in Materials and Methods. Individual optical sections of Cy5 (blue, surface HA11), Cy3 (red, internalized HA11), Cy5/Cy3 merge and Alexa-350 fluorescence $(\mathrm{CHC})$ are shown. Scale bar, $10 \mu \mathrm{m}$. $\boldsymbol{B}-\boldsymbol{D}$, PAE cells expressing YFP-HA- $\Delta$ N-DAT were incubated with Tfn-TxR (B) or LysoTrackerRed (LysoTracker) (D) for $30 \mathrm{~min}$ at $37^{\circ} \mathrm{C}$ to mark early/recycling endosomes or late endosomes/lysosomes, respectively. Fixed cells were imaged. Alternatively, the cells were fixed, permeabilized, and stained with EEA.1 antibody, followed by secondary Cy3 conjugated antibody (C). Yellow on merged images designates colocalization of YFP-HA- $\Delta$ N-DAT and Tfn-TxR (B), EEA.1 (C), or LysoTrackerRed (D) in endosomes. Optical sections through the middle of the cells are presented. Insets represent high magnification of YFP, Cy3, TexasRed, or LysotrackerRed (all Cy3 filter channel) and merged images of the regions indicated by white rectangles. Scale bars, $10 \mu \mathrm{m}$.

substituted by alanines (Fig. 5A). Our general strategy was to initially screen YFP-HA-DAT mutants for their subcellular localization and endocytic rates using the HA11 endocytosis assay in transient transfection experiments. Subsequently, PAE cell lines stably expressing the most interesting mutants (those that visibly increased endocytosis, compared with the full-length YFP-HA-

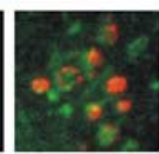

DAT) were generated and used for quantitative analysis of HA11 endocytosis. Furthermore, to avoid the effects of clonal variability, we analyzed at least two singlecell clones that expressed the same mutant or pools of cells that stably expressed the transfected DAT mutants. Deletion of the first $36 \mathrm{~N}$-terminal residues encompassing three ubiquitination sites, which are critical for PKC-dependent endocytosis of DAT, did not result in endosomal accumulation of HA11/YFP or cause any significant downregulation of surface transporter (Cy5 fluorescence) in either the absence (data not shown) or presence of monensin, compared with the full-length YFP-HA-DAT (Fig. 5B). This result suggested that the endocytosis-inhibitory signal is located within residues $37-65$. Several continuous stretches of 6 aa were then simultaneously mutated to alanines in this region of the $\mathrm{N}$-terminal tail of the fulllength YFP-HA-DAT (Fig. 5A). Examination of endocytosis of the resulting YFP-HADAT mutants revealed that only the multialanine substitution of residues $60-65$ resulted in significant accumulation of the DAT mutant (60A65 mutant) in endosomes and a corresponding decrease in the Cy5 (surface) staining of HA11 in cells treated with monensin (Fig. 5B).

The ratio of the internalized (Cy3) and surface (Cy5) fluorescence was quantified using three-dimensional imaging of cells either not treated or treated with monen$\sin$ (Fig. 5C). These quantifications demonstrated that the 60A65 mutant was constitutively internalized at a rate that was slightly lower than, but comparable with, the rate of internalization of the YFP-HA$\Delta \mathrm{N}$-DAT mutant. Thus, the data in Figure 5 suggest that residues $60-65$ are critical for the negative regulation of constitutive endocytosis by the $\mathrm{N}$-terminal tail of DAT.

\section{Relationship of DAT outward-facing conformation and plasma membrane retention}

Based on the comparative analysis of the crystal structure of the homologous bacterial leucine transporter $\left(\mathrm{LeuT}_{4}\right)$, the positively charged Arg60 proximal to the first transmembrane domain of DAT has been implicated in the regulation of DAT activity (Kniazeff et al., 2008). This conserved residue is proposed to be necessary for the outward facing conformation of the transporter by interacting with the negatively charged Asp436 in intracellular loop 4. Based on the $\mathrm{LeuT}_{4}$ structure (Yamashita et al., 2005), another residue within the region 60-65, Trp63 (homologues to Trp8 of LeuT), may also participate in the stabilization of the outward conformation by interacting with the hydrophobic residues proximal to transmembrane domain 6 . Thus, we 
hypothesized that there might be an inverse relationship between this DAT conformation and constitutive endocytosis. Kinetic measurements of $\left[{ }^{3} \mathrm{H}\right] \mathrm{DA}$ uptake by the YFP-HA- $\Delta$ N-DAT and 60A65 mutants revealed no activity of these mutants (Fig. 6A), despite comparable overall expression of both mutants and wild-type, full-length YFP-HA-DAT in these stably expressing clonal cell lines (Fig. 6B). Likewise, YFP-HA-DAT mutants in which Arg60, Trp63, or Asp436 were substituted by alanines in the full-length YFP-HADAT did not efficiently transport $\left[{ }^{3} \mathrm{H}\right] \mathrm{DA}$ into the cells (Fig. 6A). At the same time, the same mutations did not accelerate endocytosis of YFP-HA-DAT, as measured by the HA11 uptake assay (Fig. $7 A, B$ ). These data suggest that disruption of the DAT conformation by single mutations may be necessary, but not sufficient, to allow rapid constitutive endocytosis of DAT.

Surprisingly, combination of the R60A mutation and mutation of the juxtamembrane lysine (Lys65) (R60A/K65A mutant) resulted in an $\sim 2$-fold increase in internalization of HA11:DAT complexes and a substantial downregulation of surface HA11:DAT complexes in the presence of monensin, compared with the full-length, wild-type YFP-HA-DAT (Fig. 7 $A, B$ ). Interestingly, the K65A mutation alone did not significantly affect DAT-mediated uptake activity (Fig. 6) or endocytosis (Fig. $7 A, B)$. Comparative analysis of many single-cell HA11 uptake experiments in PAE cells, exemplified in Figure $7 B$, demonstrated that the enhanced endocytosis of the R60A/K65A mutant was comparable with that of the 60A65 mutant, although in all experiments there was a trend for lesser accumulation of this mutant than the 60A65 mutant in endosomes in the presence of monensin.

To confirm the results of the single-cell microscopy assay for endocytosis, the endocytic rates of wild-type full-length YFPHA-DAT and mutant DATs were estimated using an independent whole-cell population assay. In this assay, we took advantage of the observation that another HA antibody, 12CA5, binds the HA epitope in extracellular loop 2 of DAT with a lesser affinity, compared with HA11. 12CA5 can be stripped from the surface YFP-HA-DATs by treatment with acetate buffer, $\mathrm{pH}$ 2.8, thus allowing separation of the noninternalized from the internalized YFP-HA-DAT complexes using radiolabeled ${ }^{125} \mathrm{I}-12 \mathrm{AC} 5$. As shown in Figure $7 C$, the ratio of internalized to surface ${ }^{125} \mathrm{I}$ 12CA5 was considerably increased in cells expressing the YFPHA- $\Delta$ N-DAT and 60A65 mutants, compared with the ratio measured for the wild-type, full-length YFP-HA-DAT. This was true with or without monensin treatment. As in the single-cell assay, alanine substitutions of Arg60, Trp63 and Lys65 individually did not have any effect on endocytosis of DAT (data not shown),
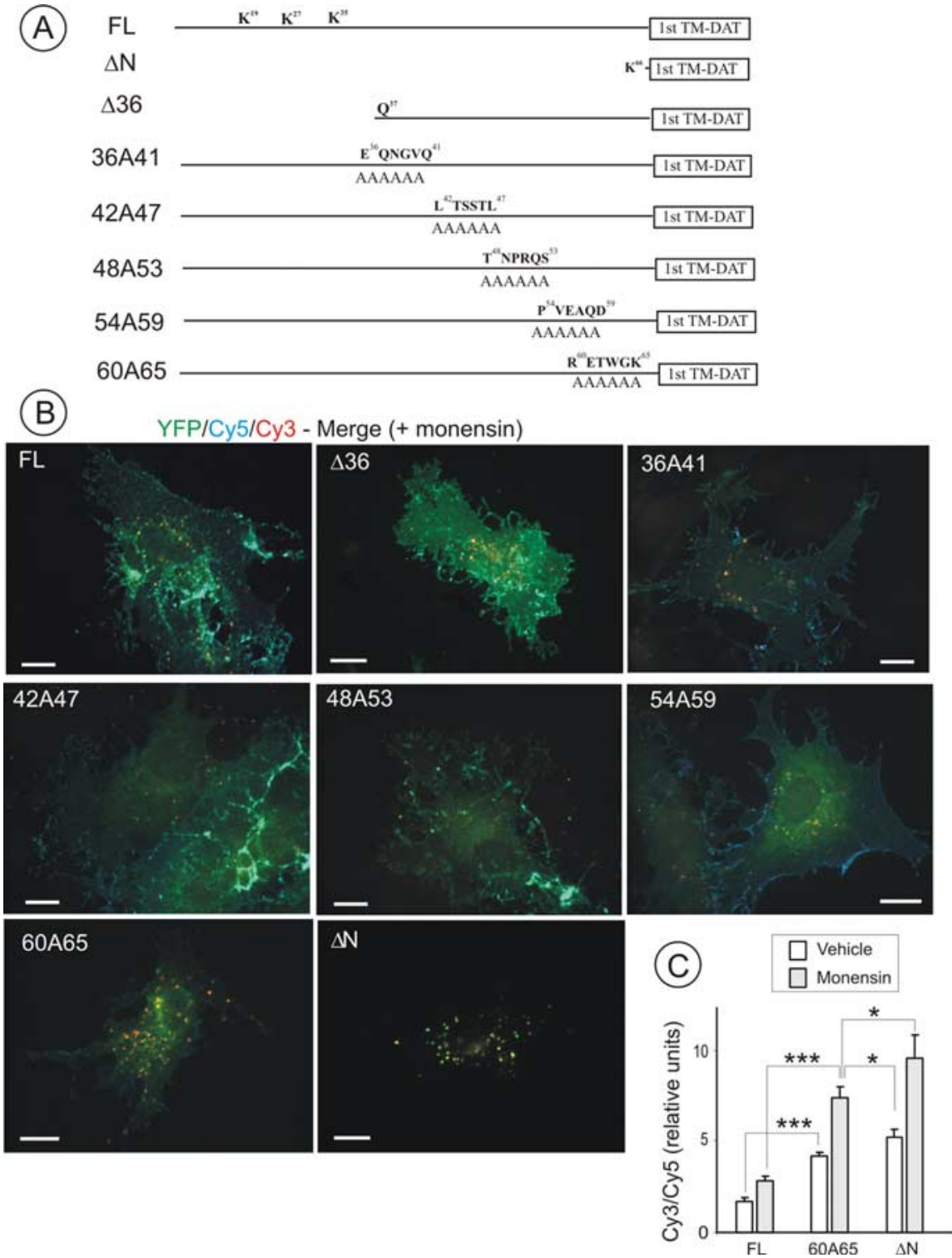

Figure 5. Residues $60-65$ are important for the endocytosis-inhibitory function of the DAT N terminus. $A$, Schematic representation of the full-length YFP-HA-DAT (FL), YFP-HA- $\Delta N-D A T(\Delta N)$, YFP-HA- $\Delta 36-D A T(\Delta 36)$, and several YFP-HA-DAT mutants in which stretches of the six amino acids indicated were substituted by alanines. $B$, PAE cells stably expressing FL or mutants of YFP-HA-DAT depicted in $\boldsymbol{A}$ were incubated with HA11 and monensin (25 $\mu \mathrm{m})$ as in Figure 3 and then stained with secondary (blue), and Cy3 (red) fluorescence are shown. Scale bars, $10 \mu \mathrm{m}$. C, Quantification of the endocytosis rates (Cy3/Cy5 ratio) of the FL YFP-HA-DAT, 60A65 mutant, and YFP-HA- $\Delta \mathrm{N}-\mathrm{DAT}$ from the experiments presented in $\boldsymbol{B}$. The averaged Cy $3 / \mathrm{Cy} 5$ ratios of internalized/surface HA11 ( \pm SEM) were calculated from at least eight cells for each cell line. ${ }^{*} p<0.05 ;{ }^{* * *} p<0.001$ (unpaired $t$ test), 60A65 compared with the corresponding data in FL- and YFP-HA- $\Delta \mathrm{N}-\mathrm{DAT}$-expressing cells.

whereas a double mutant, R60A/K65A, was internalized at an increased rate with or without monensin treatment (Fig. 7C). The endocytosis of the D436A mutant was somewhat reduced compared with that of the full-length DAT.

The data in Figure 7 suggest that disruption of the DAT conformation is not sufficient to promote rapid endocytosis of DAT; but when this is combined with the K65A mutation, it may yield an accelerated endocytosis phenotype, as observed with the R60A/K65A mutant. To further test this hypothesis, the K65A mutation was combined with other mutations that disrupt the outward facing conformation of DAT (Fig. 8A). W63A or D436A mutations combined with K65A (63A65 and K65A/D436A mutants) resulted in increased endocytosis, compared with corre- 
(A)

\begin{tabular}{|c|c|c|}
\hline Cell line & $\mathrm{Km}(\mu \mathrm{M})$ & $\begin{array}{l}\text { Vmax } \\
(\% \mathrm{FL})\end{array}$ \\
\hline $\mathrm{FL}$ & $2.46 \pm 0.87$ & 100 \\
\hline$\Delta \mathrm{N}$ & N.D. & $0.1 \pm 0.1$ \\
\hline $60 A 65$ & N.D. & N.D. \\
\hline $\mathrm{R} 60 \mathrm{~A}$ & N.D. & N.D. \\
\hline W63A & N.D. & N.D. \\
\hline K65A & $1.40 \pm 0.91$ & $90.9 \pm 6.7$ \\
\hline R60A/K65A & N.D. & $0.5 \pm 0.1$ \\
\hline $63 A 65$ & N.D. & N.D. \\
\hline RWK/AAA & N.D. & N.D. \\
\hline D436A & $0.06 \pm 0.00$ & $8.4 \pm 1.6$ \\
\hline
\end{tabular}

N.D., Not Detectable

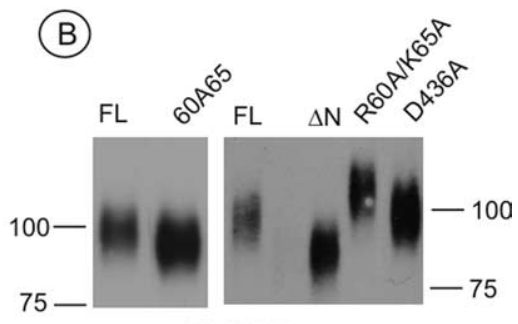

IP: HA11

WB: HA11

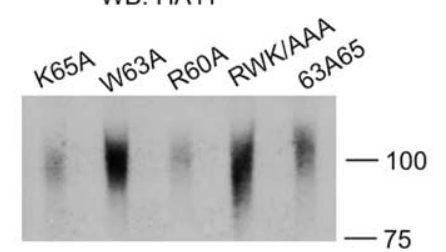

IP: HA11

WB: anti-DAT

Figure 6. $\left[{ }^{3} \mathrm{H}\right] \mathrm{DA}$ uptake by YFP-HA-DAT mutants. $A,\left[{ }^{3} \mathrm{H}\right] \mathrm{DA}$ uptake kinetics were measured in PAE cells stably expressing full-length (FL) or YFP-HA-DAT mutants, and the resulting parameters [affinity $(\mathrm{Km})$ and maximal velocity $\left(V_{\text {max }}\right) ; \pm \mathrm{SEM}, N=$ $2-5$ ] are shown. $V_{\max }$ values were normalized to the relative amount of the transporter immunoprecipitated with HA11 (described in $\boldsymbol{B}$ ) and expressed as percentage of the normalized $V_{\text {max }}$ value obtained in the FL YFP-HA-DAT-expressing cells. $\boldsymbol{B}$, Various cell clones expressing DAT mutants (top blot) or pools of stably expressing cells (bottom blot) were incubated with HA11 antibodies for $90 \mathrm{~min}$ at $37^{\circ} \mathrm{C}$; and after solubilization, HA11:YFP-HA-DAT complexes were precipitated using Protein G-Sepharose followed by electrophoresis. DAT immunoreactivity was detected by blotting with HA11 or DAT antibodies. Note an abnormal slow mobility on the gel of the R60A/K65A mutant that was consistently observed.

sponding single mutants and wild-type full-length DAT (Fig. $8 B, C)$. The RWK/AAA mutant displayed an accelerated constitutive endocytosis when measured in the presence of monensin, resulting in an endocytic rate that exceeds that of the $60 \mathrm{~A} 65 \mathrm{mu}-$ tant (Fig. 8C). Furthermore, simultaneous mutations of residues 60-62 to alanines did not change the endocytic trafficking of YFP-HA-DAT (data not shown). Altogether, the data from the DAT mutagenesis studies presented in Figures 7 and 8 suggest that residues important for the outward facing conformation of DAT cooperate with Lys65 in retaining DAT at the cell surface and/or preventing rapid constitutive endocytosis of DAT.

\section{Residues important for DAT uptake activity are also involved in regulation of the localization of DATs in membrane protrusions and the mobile fraction of DATs}

Visual inspection of numerous images of the YFP and Cy5 fluorescence of YFP-HA-DATs after the HA11 endocytosis assay revealed a dramatic difference in the distribution of wild-type and mutant DATs in the plasma membrane. Most of cells expressing wild-type DAT displayed a substantial concentration of the transporter in membrane protrusions (or filopodia) and cell edges, resulting in a characteristic "contoured" phenotype (Fig. 9A). Filopodia accumulation of YFP-HA-DAT was observed in PAE (Fig. 9A), HeLa and HEK293 cells (data not shown), as well as in axonal widenings and large somas of embryonic dopaminergic neurons (data not shown). In contrast, all mutants with a disrupted outward-facing conformation were distributed evenly throughout the different macrostructures of the plasma membrane, including actin-rich filopodia, lamellopodia and ruffles (Fig. 9A). The K65A mutant displayed a plasma membrane distribution identical to that of wild-type YFP-HA-DAT. Because sites of actin polymerization in the plasma membrane are known to trap transmembrane proteins and decrease their mobility, the lateral mobility of DAT mutants was measured using the FRAP method. In these experiments, a mobile fraction $\left(M_{\mathrm{f}}\right)$ and diffusion time $\left(\tau_{\mathrm{D}}\right)$ (related to the diffusion coefficient $D_{\tau}$ ) were mea-

sured in individual filopodia, ruffles, and areas of diffuse distribution of HA-YFPDATs at the periphery of the cells (Fig. 9B). The $M_{\mathrm{f}}$ value for the full-length YFP-HADAT in filopodia/ruffles was half that in the diffuse areas (Fig. $9 C$ ), whereas $\tau_{\mathrm{D}}$ values were similar (Fig. 9D). Similar results were obtained with the K65A mutant (Fig. $9 C, D)$. Although the accumulation of the other mutants in filopodia and cell contours was very rarely observed, the $M_{\mathrm{f}}$ values for the other DAT mutants in these structures were also low (Fig. 9C). The exception was the cells expressing YFP-HA$\Delta \mathrm{N}$-DAT where we found no examples of structures with slow mobility of this mutant transporter. Combining the FRAP data from all mutants, filopodia had the lowest values of $M_{\mathrm{f}}(\sim 25 \%)$, whereas this fraction was higher in ruffles $(\sim 40 \%)$ and highest in areas of diffuse distribution of YFP fluorescence ( $60 \%)$ (Fig. $9 E$ ). There were no statistically significant differences in $\tau_{\mathrm{D}}$ values measured for any of the mutants in all membrane areas. The averaged value for the lateral diffusion coefficient $D_{\tau}$ was $0.3-0.4 \times 10^{-9} \mathrm{~cm}^{2} / \mathrm{s}$, which is well within the range of the typical $D_{\tau}$ values of integral membrane proteins, including DAT (Adkins et al., 2007). Altogether, the data suggest that mutations resulting in the disruption of the outward-facing conformation of DAT and necessary for elevated constitutive endocytosis of the transporter also lead to a loss of the filopodia targeting of DAT and therefore result in an increased plasma membrane pool of mobile DATs.

\section{Effect of AMPH on DAT localization and endocytosis}

AMPH treatment of cells results in "reversal" of DATs leading to the substrate efflux, presumably, by shifting the transporter from the outward- to inward-facing conformation (Khoshbouei et al., 2004; Fog et al., 2006). It is, therefore, possible that AMPH has an effect on DAT conformation that is similar to the effects of mutations that result in the disruption of the outward-facing conformation of DAT (R60A, W63A or D436A), and that combining AMPH exposure with the K65A mutation may have the same impact on DAT endocytosis as did the combination of R60A, W63A or D436A mutations with the K65A mutation (Fig. 8). To test this hypothesis, the effects of AMPH $(20 \mu \mathrm{M})$ pretreatment on localization and endocytosis of the wild-type YFP-HA-DAT and the K65A mutant expressed in PAE cells were compared using the HA11 endocytosis assay.

Analysis of the surface distribution of YFP-HA-DAT (Cy5 fluorescence) revealed that AMPH dramatically decreased the accumulation of the transporter in filopodia and led to a more equal distribution of HA11 immunoreactivity within the cell edges, ruffles and flat areas of the plasma membrane (Fig. 10A). The latter effect was evident from the significant decrease in the fluctuation of the pixel-by-pixel Cy5 fluorescence intensity in AMPH-treated cells compared with vehicle-treated cells (supplemental Fig. 1, available at www.jneurosci.org as supplemental material). The loss of filopodia localization of YFP-HA-DAT was typically observed in $70-80 \%$ of cells after 30 -min incubation with AMPH and reached a maximum after a 60-min AMPH exposure. Essentially identical redistribution of the K65A mutant 


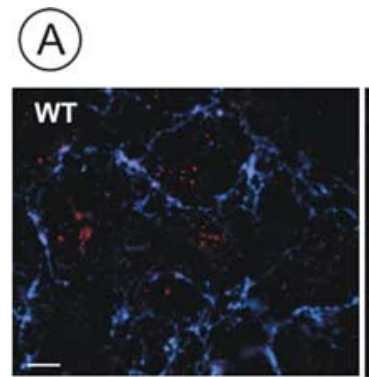

\section{Cy5 (surface) / Cy3 (internalized)}
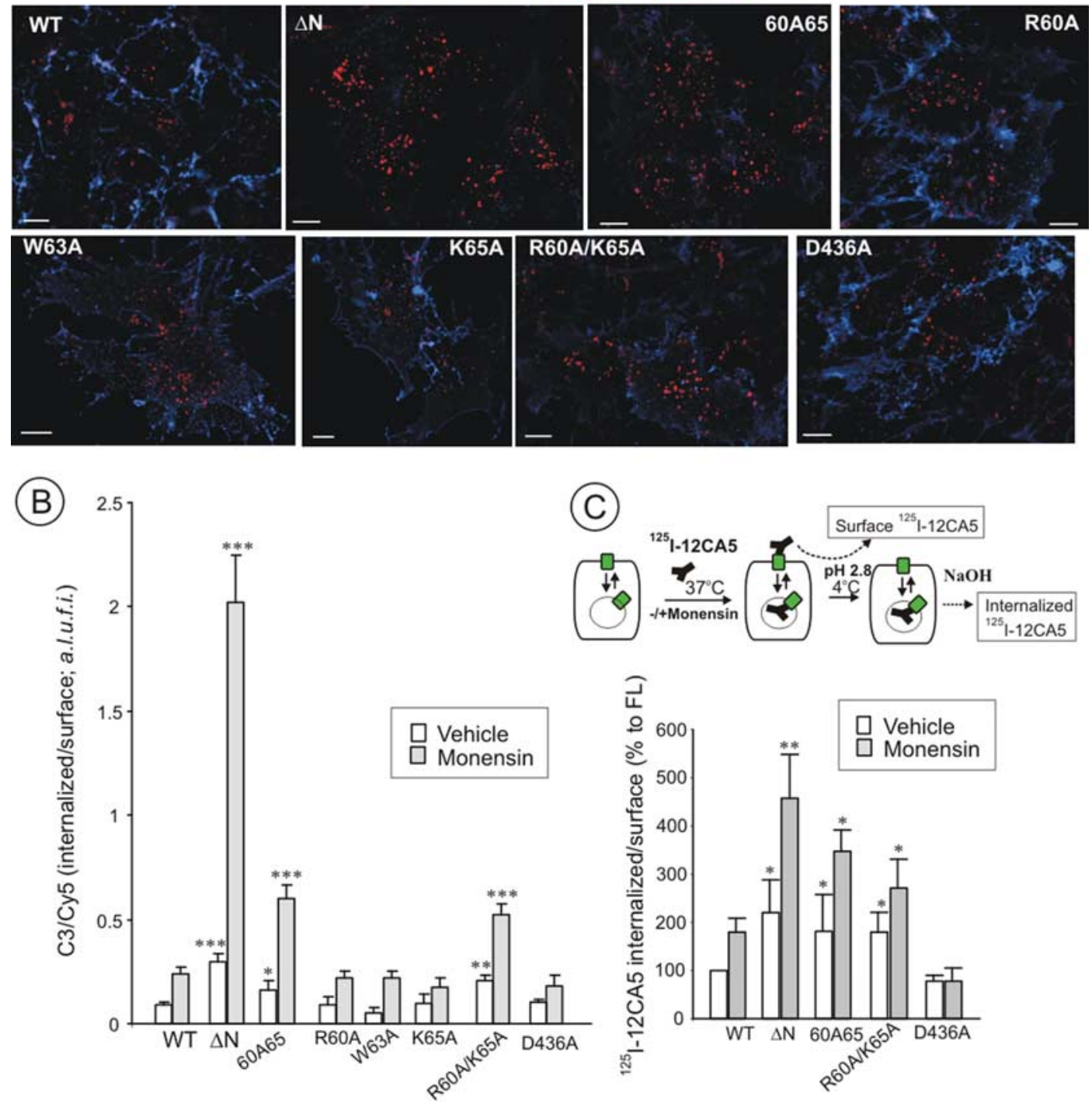

Figure 7. Constitutive endocytosis of single and double amino acid mutants of YFP-HA-DAT.A, An HA11 endocytosis assay was performed in PAE cells stably expressing full-length wild-type (WT), YFP-HA- $\Delta \mathrm{N}-\mathrm{DAT}(\Delta \mathrm{N})$, or various mutants of full-length YFP-HA-DAT. The cells were incubated with $25 \mu \mathrm{m}$ monensin for the last 30 min of incubation with HA11 as described in Figure 3 . Merged images of Cy5 (blue) and Cy3 (red) fluorescence are presented. Individual optical sections are shown. Scale bars, $10 \mu \mathrm{m}$. B, Quantification of the endocytosis rates (Cy3/Cy5 ratio) of the WT and mutant YFP-HA-DATs from the experiments presented in $A$. The cells were either not treated or treated with $25 \mu \mathrm{m}$ monensin. Averaged Cy3/Cy5 ratios of internalized/surface HA11 ( \pm SEM) were calculated from at least 20 cells for each cell line. ${ }^{*} p<0.05 ;{ }^{* *} p<0.005 ;{ }^{* * *} p<0.001$ (unpaired $t$ test), compared with the corresponding data in WT-expressing cells. C, Uptake of ${ }^{125}$ I-12CA5 antibody into PAE cells stably expressing WT or mutant YFP-HA-DATs. The diagram depicts the steps of the experimental protocol described in Materials and Methods. The cells were incubated with radiolabeled antibodies for $60 \mathrm{~min}$ and then for additional $30 \mathrm{~min}$ in either the absence (vehicle) or the presence of monensin. The noninternalized fraction of ${ }^{125} \mathrm{I}-12 \mathrm{CA} 5 \mathrm{was}$ removed by an acid wash, whereas the internalized fraction was obtained by solubilization of the acid-washed cells. The ratios of internalized to surface ${ }^{125}$ - 12 CA5 are presented as a percentage of this ratio in WT-YFP-HA-DAT-expressing cells ( \pm SEM) calculated from at least 20 cells for each cell line. ${ }^{*} p<0.05 ;{ }^{* *} p<0.005$ (unpaired $t$ test), compared with the corresponding data in WT-expressing cells.

from filopodia was observed (Fig. 10B). The pattern of the plasma membrane localization of YFP-HA-DAT in AMPHtreated cells was very similar to that observed for YFP-HA-DAT mutants with disrupted outward-facing conformation (R60A, W63A or D436A) (Fig. 9).

Quantification of the mean intensity of surface YFP-HA-DAT fluorescence (Cy5) showed that AMPH resulted in $\sim 20-25 \%$ downregulation of the surface transporter levels, presumably due to acceleration of DAT endocytosis. Measurements of the Cy3/ Cy5 ratios confirmed that AMPH accelerated DAT endocytosis in both wild-type and mutant-expressing cells (Fig. $10 B, C$ ), although the AMPH-induced internalization was significantly slower than in the case of cell stimulation with phorbol esters (data not shown). Interestingly, the extent of the AMPH-induced endocytosis of the K65A mutant was larger (3-fold increase in the Cy3/Cy5 ratio) than that of the wild-type YFP-HA-DAT (2-fold increase in the $\mathrm{Cy} 3 / \mathrm{Cy} 5$ ratio) (Fig. 10C). Altogether, the data in Figure 10 support the hypothesis that AMPH exposure affects DAT conformation and trafficking in manner that is similar to the effects of mutations disrupting the outward-facing conformation of the transporter.

\section{Discussion}

Regulation of the number of DATs present at the surface of dopaminergic neurons plays an important role in controlling the strength and duration of DA neurotransmission in the brain. In 
(A)

$\begin{array}{ll}\text { WT } & \mathbf{R}^{60} \text { ETWGK }^{65} \\ \text { 60A65 } & A^{60} \text { AAAAA }^{65} \\ \text { 63A65 } & \mathbf{R}^{60} \text { ETAAA }^{65} \\ \text { RWK/AAA } & \text { A }^{60} \text { ETAGA }^{65} \\ \text { K65A/D436A } & \mathbf{R}^{60} \text { ETWGA }^{65}-A^{436}\end{array}$

(B)

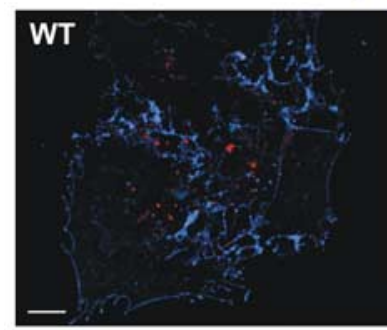

Cy5 (surface) / Cy3 (internalized)
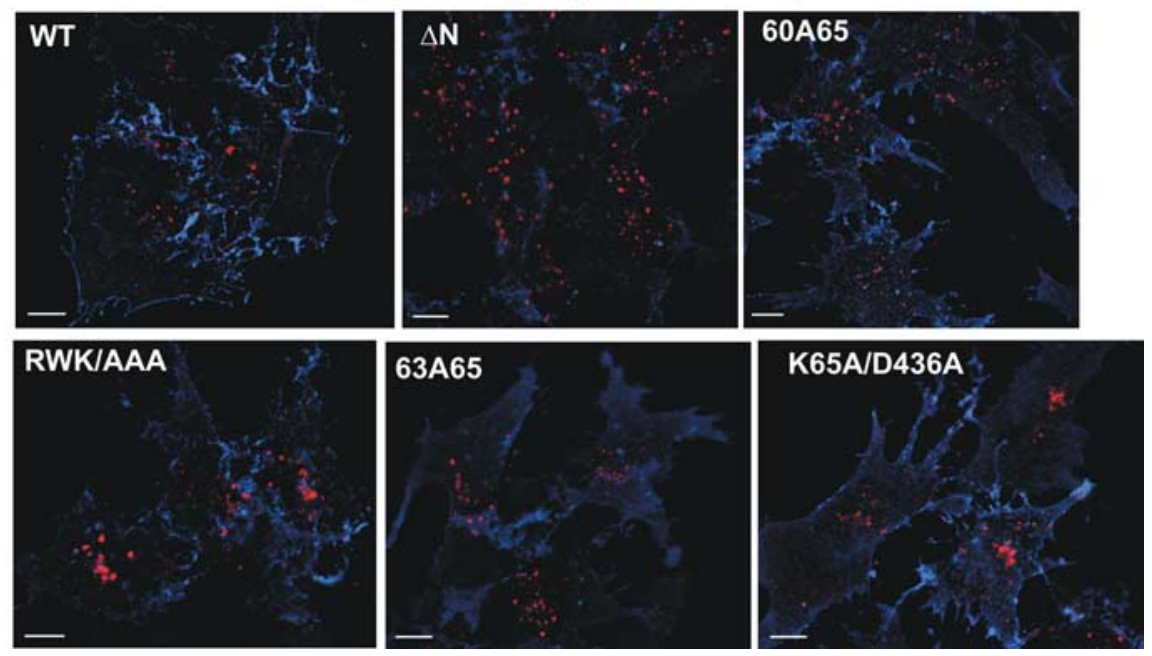

Figure 8. Constitutive endocytosis of HA-YFP-DAT mutants with combinations of several single amino acid mutations within the region $60-65$. $A$, Schematic representation of YFP-HA-DAT mutants with multiple mutations in the $60-65$ region. $B, P A E$ cells stably expressing full-length wild-type (WT) or mutants of YFP-HA-DAT depicted in $\boldsymbol{A}$ were incubated with HA11 and monensin $(25 \mu \mathrm{m})$ as in Figure 3 and then stained with secondary antibodies as described in Materials and Methods. Individual optical sections are presented. Merged images of Cy5 (blue) and Cy3 (red) fluorescence are shown. Scale bars, $10 \mu \mathrm{m}$. C, Quantification of the endocytosis rates (Cy3/Cy5 ratio) of the WT and mutant YFP-HA-DAT from experiments similar to those presented in $\boldsymbol{B}$. The averaged Cy3/Cy5 ratios of internalized/surface HA11 ( \pm SEM) were calculated from at least 20 cells for each cell clone. ${ }^{*} p<0.05$ (unpaired $t$ test), RWK/AAA mutant compared with the 60 A65 mutant.

this study we found that the intracellular N-terminal tail of DAT has an inhibitory influence on its constitutive internalization, thus enhancing retention of DATs on the cell surface and DATmediated uptake activity. Our findings contribute to the understanding of the multiple roles played by the $\mathrm{N}$ terminus in regulating DAT function, such as the requirement of DAT N-terminal phosphorylation for AMPH-induced efflux (Khoshbouei et al., 2004) and maintaining the outward-facing conformation of the transporter necessary for the substrate uptake (Kniazeff et al., 2008). The $\mathrm{N}$ terminus is also necessary for the delivery of newly synthesized DATs to the plasma membrane, as demonstrated by intracellular accumulation of a DAT mutant lacking the first 60 residues (Torres et al., 2003). However, our internalization experiments were possible because we found that the replacement of the $\mathrm{N}$ terminus by YFP or a tandem of $10 \times$ His- and Flag-tags rescues the inefficient plasma membrane delivery of the $\Delta \mathrm{N}-\mathrm{DAT}$.

A single-cell internalization assay developed in our previous studies (Sorkina et al., 2006) was the main method used to measure the endocytic rates in the present study. In addition, a new whole-population biochemical assay using the radiolabeled HA 12CA5 antibody was developed to complement the single-cell assay (Fig. 7C). In general, both endocytic assays using the different HA antibodies yielded similar results. However, the assay with the 12CA5 antibody had limitations in measuring the endocytic rates in cells expressing relatively low levels of YFPHA-DAT mutants because of the low signal-to-noise ratio, which is due to the weak affinity of this antibody for the internally inserted HA epitope.

Analysis of YFP-HA- $\Delta$ N-DAT endocytosis in neurons suggested that this mutant traffics in a manner similar to its trafficking in PAE and HeLa cells, and that the $\mathrm{N}$ terminus has the same endocytosisinhibitory role in neurons (Fig. 2). Although it is difficult to quantitatively compare neurons and non-neuronal cells due to low efficiency of transfection of neurons, the current experiments with HA11 antibody (Fig. 2) and our previous observations (Sorkina et al., 2006) suggest that wild-type DAT is accumulated in endosomes to a larger extent in embryonic neurons at steady-state growth conditions than in non-neuronal expression systems, such as PAE, HEK293, $1 \mathrm{RB}_{3} \mathrm{AN}_{27}$ and HeLa cells. Such endosomal accumulation could be due to increased internalization or reduced recycling of DAT compared with non-neuronal cells. Furthermore, it remains to be determined in future studies whether the N-terminal domain of DAT has an endocytosis-inhibitory role in adult dopaminergic neurons. The existence of such mechanisms that prevent rapid constitutive endocytosis of DAT are likely important for regulation of DA neurotransmission in the brain because DAT functions at the plasma membrane.

Mutagenesis of the N-terminal tail of DAT revealed that the conserved amino acid residues $60-65$ play a key role in the inhibitory function of this domain of the DAT molecule. We propose a model whereby a positively charged juxtamembrane Lys65 cooperates with the intramolecular interactions (involving Arg60, Trp63 and Asp436) responsible for maintaining the outward conformation of DAT and thereby retains DAT at the cell surface and/or prevents its rapid constitutive endocytosis. Interestingly, the RWK/ AAA mutant was internalized slightly faster than the 60A65 mutant (Fig. 8). It is possible that mutation of the negatively charged residue Glu61 somewhat counteracted the elimination of the positive charge of Arg60 within the interaction network involving the 60-65 motif. It should be noted that other parts of the $\mathrm{N}$ terminus may have additional contribution(s) in the constitutive accumulation of DATs in endosomes because none of the sitepoint mutants in the region $60-65$, including the RWK/AAA mutant, fully reproduced the dramatic endocytic phenotype of YFP-HA- $\Delta$ N-DAT.

What could be the mechanisms by which Lys65 and residues important for the DAT conformation negatively regulate the constitutive endocytosis of DATs? It is likely that the mechanisms mediating constitutive internalization of full-length YFP-HADAT and YFP-HA- $\Delta$ N-DAT are similar as they both use the clathrin-coated pit pathway (Fig. 4) (Sorkina et al., 2005). One possibility is that the release of the intramolecular interactions 

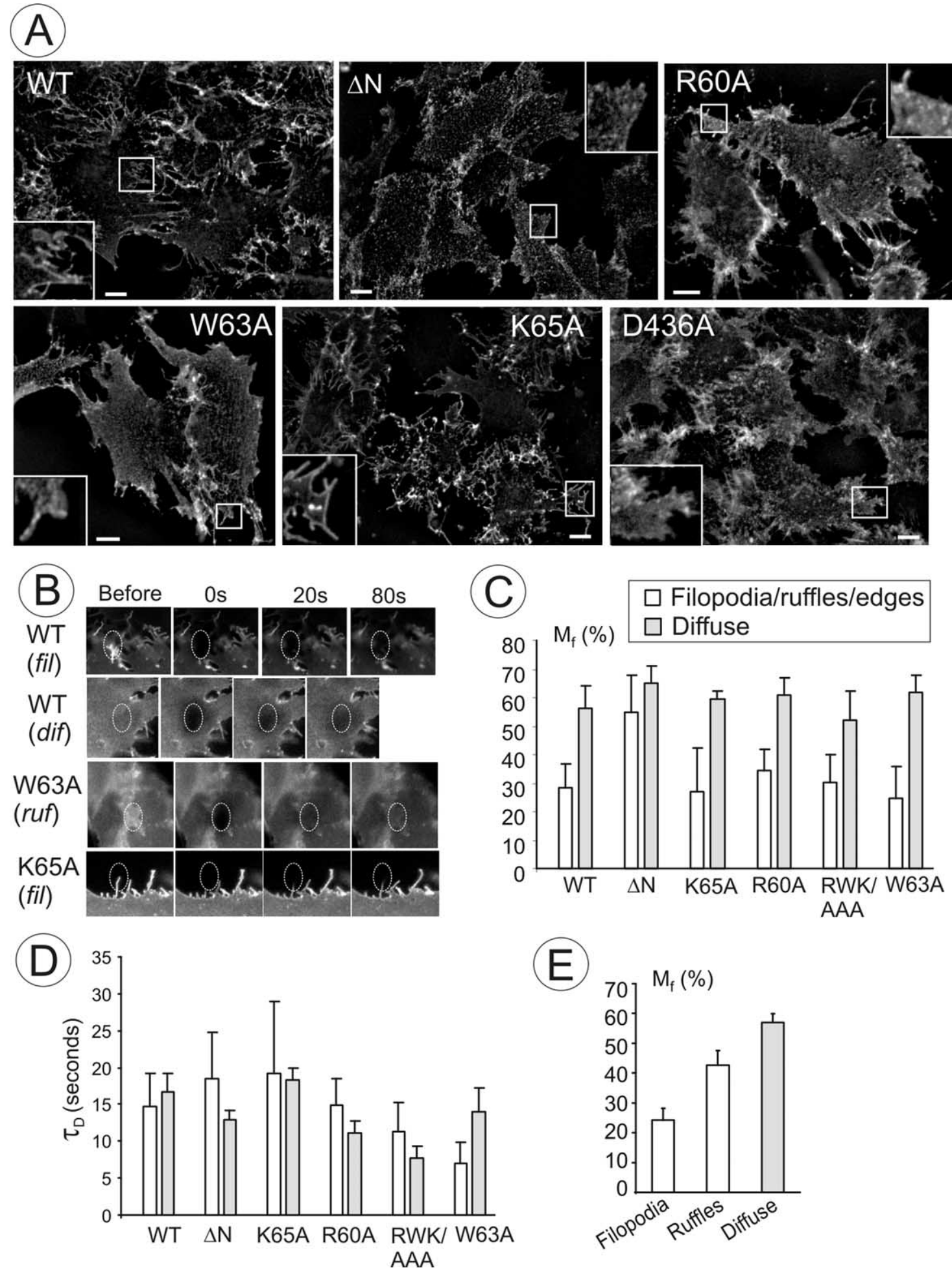

Figure 9. Distribution and lateral mobility of YFP-HA-DATs in the plasma membrane of PAE cells. A, Cells expressing full-length wild-type (WT) and mutant YFP-HA-DATs were subjected to an HA11 endocytosis assay as described in Figure 1. Optical sections of Cy5 fluorescence through the plane of the plasma membrane are shown. Insets represent high-magnification images of the regions indicated by white rectangles. Scale bars, $10 \mu \mathrm{m}$. B, Examples of images obtained in FRAP experiments before (Before) or 0, 20, and 80 s after photobleaching of filopodia (fil), ruffles (ruf), or diffuse areas of fluorescence (dif). The bleach areas are indicated by dashed ovals. $\boldsymbol{C}, \boldsymbol{D}$, Mobile fraction $\left(M_{\mathrm{f}}\right)$ and diffusion time $\left(\tau_{\mathrm{D}}\right)$ of WT and mutant YFP-HA-DATs were measured in FRAP experiments as described in Materials and Methods. Mean values from three to five cells ( \pm SEM) were obtained for filopodia, ruffles, and cell edge areas or from areas of diffuse YFP fluorescence in the periphery of the cells. $\boldsymbol{E}$, Mean values of $M_{\mathrm{f}}( \pm \mathrm{SEM})$ combined from all cell lines were averaged for filopodia (Filopodia), ruffled membranes (Ruffles), and areas of the diffuse YFP fluorescence (Diffuse).

supporting the outward conformation exposes a "positive" internalization signal, but that rapid endocytosis is prevented by additional interactions at the plasma membrane, presumably involving Lys65. Another possibility is that the disruption of the outward DAT conformation leads to the release of DATs retained at the plasma membrane. The existence of a pool of immobile DATs that is located in membrane protrusions (or filopodia) and sensitive to mutation disrupting DAT activity suggested that res- 



Figure 10. Effects of AMPH on plasma membrane distribution and endocytosis of the wild-type HA-YFP-DAT and K65A mutant. PAE cells stably expressing full-length wild-type (WT) or K65A mutants of YFP-HA-DAT were incubated with HA11 for $1 \mathrm{~h}$ at $37^{\circ} \mathrm{C}$ in $\mathrm{KRH}$, and then AMPH $(20 \mu \mathrm{m})$ was added and the cells were further incubated for 30 or $60 \mathrm{~min}$ at $37^{\circ} \mathrm{C}$. After fixation, the cells were stained with secondary antibodies as described in Materials and Methods. A, Cy5 fluorescence images of WT-expressing cells incubated with vehicle or AMPH for $60 \mathrm{~min}$ are shown. Individual optical sections through the plasma membrane (maximum Cy5 fluorescence) are presented. Note the large intensity scale in the Veh image. Scale bars, $10 \mu \mathrm{m}$. B, Merged images of Cy5 (blue) and Cy3 (red) fluorescence of cells treated with vehicle or AMPH for $30 \mathrm{~min}$ are shown. Individual optical sections through the middle of the cells are presented. Scale bars, $10 \mu \mathrm{m}$. C, Quantification of the endocytosis rates (Cy3/Cy5 ratio) of the WT and K65A mutant YFP-HA-DAT. The averaged Cy3/Cy5 ratios of internalized/surface HA11 ( \pm SEM) were calculated from at least 20 cells for each condition. ${ }^{*} p<0.05$.

idues supporting the DAT outward conformation are also important for targeting of DAT to filopodia (Fig. 9). Accumulation in filopodia has been demonstrated previously to prevent clathrindependent endocytosis of transmembrane proteins (Hommelgaard et al., 2004). Interestingly, the pattern of localization of the wild-type YFP-HA-DAT and the K65A mutant was very similar to that of the lipid phosphatase related protein 1, a transmembrane protein that is localized in filopodia and that can induce formation of filopodia when overexpressed (Sigal et al., 2007). Filopodia that are broadly defined as slender membrane protrusions containing a core of bundled actin filaments play important roles in signaling, cell migration and attachment, and neurite outgrowth in neurons (Wood and Martin, 2002; Kalil and Dent, 2005). We did not detect obvious differences in the relative distribution of the full-length YFP-HADAT and YFP-HA- $\Delta$ N-DAT between different compartments of embryonic neurons, suggesting that the N-terminal domain is not essential for targeting of the transporter to axonal or somatodendritic compartments of these neurons. However, targeting of DATs to filopodia and structures derived from filopodia could be critical for correct perisynaptic localization of DAT within axonal varicosities and distal dendrites, and during developmental maturation of dopaminergic neurons. Furthermore, restriction of the mobility of the plasma membrane pool of DATs at the synapse may be one of the mechanisms by which proper intramolecular DAT interactions prevent DATs from rapid endocytosis in neurons.

Our experiments with AMPH (Fig. 10) revealed the similarity of the effects of conformation-destabilizing mutations and AMPH on DAT localization and endocytosis. AMPH treatment leads to phosphorylation of the distal $\mathrm{N}$ terminus, which in turn leads to the reverse substrate transport, or efflux, presumably due to a switch of the DAT conformation to inward-facing (Khoshbouei et al., 2004; Fog et al., 2006). The molecular mechanism by which phosphorylation of the $\mathrm{N}$ terminus leads to conformational changes within the functional core of the transporter molecule is unknown. The similarity of the effects of mutations such as R60A, W63A and D436A (Fig. 9) and AMPH (Fig. 10) on the plasma membrane distribution of DAT support the hypothesis that AMPH treatment disrupts the outward-facing conformation of DAT. This observation also supports the hypothesis that this conformation is necessary for filopodia localization and presence of an immobile fraction of DAT. Moreover, the K65A mutation potentiated the endocytosis-accelerating effect of AMPH, further indicating the similarity of the effects of AMPH and conformationdisrupting mutations. However, in contrast to these mutations, AMPH alone caused an acceleration of endocytosis, suggesting the effects of AMPH are more complex. For instance, AMPHinduced activation of PKC and/or CaMKII, as well as inhibition of the Akt kinase and interaction with Syntaxin 1A may all contribute to the mechanisms responsible for endosomal accumulation of DAT in AMPH-treated cells (Johnson et al., 2005; Fog et al., 2006; Fleckenstein et al., 2007; Wei et al., 2007; Binda et al., 2008). In general, it is likely that the molecular determinants in the DAT molecule that are responsible for constitutive and induced endocytosis are different. Different residues in the C-terminal domain have been suggested to control constitutive 
and PKC-dependent endocytosis (Holton et al., 2005). Elimination of ubiquitination sites that are proposed to be involved in PKC-induced internalization either by truncation (Fig. 5) or single amino acid substitutions (Miranda and Sorkin, 2007) did not affect constitutive endocytosis of DAT.

The mechanism by which Lys65 negatively regulates DAT endocytosis remains to be defined. Because the K65A mutation does not significantly affect DAT-mediated substrate uptake activity and plasma membrane distribution of DAT, it is unlikely that K65A alone leads to significant conformational changes in the DAT molecule. However, it is possible that K65A mutation further destabilizes the outward-facing conformation or expose a positive internalization signal in the presence of other mutations. Alternatively, according to the "retention" model, Lys65 could be responsible for DAT interactions with a plasma membrane protein or other components of the plasma membrane that prevent the recruitment of DATs into clathrin-coated pits. It has been demonstrated that DATs are associated with lipid rafts and that this association is important for DAT-mediated DA uptake activity (Adkins et al., 2007; Foster et al., 2008). However, it is unlikely that Lys65 is responsible for the raft localization of DATs since mutation of this residue did not significantly affect DAT uptake activity (Fig. 6). Interestingly, whereas residues important for the outward-facing conformation of DAT are highly conserved among this transporter family, including LeuT, lysine in the position corresponding to Lys65 in DAT is not present in LeuT and is conserved only in transporters closely related to DAT, viz., the norepinephrine and serotonin transporters.

In summary, it is possible that masking of the positive internalization motif and active retention of DATs in the plasma membrane both contribute to the negative regulation of DAT endocytosis by the membrane-proximal stretch of amino acid residues in the $\mathrm{N}$ terminus of the transporter. This regulation may have important consequences for the physiological control of DAT activity. DATs can be transiently converted into an "inward-facing" state during the release of substrate (Yamashita et al., 2005) or switched to the reverse transport conformation by phosphorylation of N-terminal residues (Khoshbouei et al., 2004; Fog et al., 2006), thus facilitating DAT clearance from the cell surface by the endocytic machinery. In such cases, we propose that intramolecular and/or intermolecular interactions involving Lys65 prevent rapid endocytosis of DATs, thereby retaining transporters on the cell surface.

\section{References}

Adkins EM, Samuvel DJ, Fog JU, Eriksen J, Jayanthi LD, Vaegter CB, Ramamoorthy S, Gether U (2007) Membrane mobility and microdomain association of the dopamine transporter studied with fluorescence correlation spectroscopy and fluorescence recovery after photobleaching. Biochemistry 46:10484-10497.

Axelrod D, Koppel DE, Schlessinger J, Elson E, Webb WW (1976) Mobility measurement by analysis of fluorescence photobleaching recovery kinetics. Biophys J 16:1055-1069.

Binda F, Dipace C, Bowton E, Robertson SD, Lute BJ, Fog JU, Zhang M, Sen N, Colbran RJ, Gnegy ME, Gether U, Javitch JA, Erreger K, Galli A (2008) Syntaxin 1A interaction with the dopamine transporter promotes amphetamine-induced dopamine efflux. Mol Pharmacol 74:1101-1108.

Daniels GM, Amara SG (1999) Regulated Trafficking of the Human Dopamine Transporter. Clathrin-mediated internalization and lysosomal degradation in response to phorbol esters. J Biol Chem 274:35794-35801.

Fleckenstein AE, Volz TJ, Riddle EL, Gibb JW, Hanson GR (2007) New insights into the mechanism of action of amphetamines. Annu Rev Pharmacol Toxicol 47:681-698.

Fog JU, Khoshbouei H, Holy M, Owens WA, Vaegter CB, Sen N, Nikandrova Y, Bowton E, McMahon DG, Colbran RJ, Daws LC, Sitte HH, Javitch JA, Galli A, Gether U (2006) Calmodulin kinase II interacts with the dopa- mine transporter $\mathrm{C}$ terminus to regulate amphetamine-induced reverse transport. Neuron 51:417-429.

Foster JD, Adkins SD, Lever JR, Vaughan RA (2008) Phorbol ester induced trafficking-independent regulation and enhanced phosphorylation of the dopamine transporter associated with membrane rafts and cholesterol. J Neurochem 105:1683-1699.

Gether U, Andersen PH, Larsson OM, Schousboe A (2006) Neurotransmitter transporters: molecular function of important drug targets. Trends Pharmacol Sci 27:375-383.

Holton KL, Loder MK, Melikian HE (2005) Nonclassical, distinct endocytic signals dictate constitutive and PKC-regulated neurotransmitter transporter internalization. Nat Neurosci 8:881-888.

Hommelgaard AM, Lerdrup M, van Deurs B (2004) Association with membrane protrusions makes ErbB2 an internalization-resistant receptor Mol Biol Cell 15:1557-1567.

Johnson LA, Guptaroy B, Lund D, Shamban S, Gnegy ME (2005) Regulation of amphetamine-stimulated dopamine efflux by protein kinase $\mathrm{C}$ beta. J Biol Chem 280:10914-10919.

Kalil K, Dent EW (2005) Touch and go: guidance cues signal to the growth cone cytoskeleton. Curr Opin Neurobiol 15:521-526.

Khoshbouei H, Sen N, Guptaroy B, Johnson L, Lund D, Gnegy ME, Galli A, Javitch JA (2004) N-terminal phosphorylation of the dopamine transporter is required for amphetamine-induced efflux. PLoS Biol 2:E78.

Kniazeff J, Shi L, Loland CJ, Javitch JA, Weinstein H, Gether U (2008) An intracellular interaction network regulates conformational transitions in the dopamine transporter. J Biol Chem 283:17691-17701.

Loder MK, Melikian HE (2003) The dopamine transporter constitutively internalizes and recycles in a protein kinase $\mathrm{C}$-regulated manner in stably transfected PC12 cell lines. J Biol Chem 278:22168-22174.

Melikian HE (2004) Neurotransmitter transporter trafficking: endocytosis, recycling, and regulation. Pharmacol Ther 104:17-27.

Melikian HE, Buckley KM (1999) Membrane trafficking regulates the activity of the human dopamine transporter. J Neurosci 19:7699-7710.

Miranda M, Sorkin A (2007) Regulation of receptors and transporters by ubiquitination: new insights into surprisingly similar mechanisms. Mol Interv 7:157-167.

Miranda M, Dionne KR, Sorkina T, Sorkin A (2007) Three ubiquitin conjugation sites in the amino terminus of the dopamine transporter mediate protein kinase C-dependent endocytosis of the transporter. Mol Biol Cell 18:313-323.

Sigal YJ, Quintero OA, Cheney RE, Morris AJ (2007) Cdc42 and ARP2/3independent regulation of filopodia by an integral membrane lipidphosphatase-related protein. J Cell Sci 120:340-352.

Sorkina T, Doolen S, Galperin E, Zahniser NR, Sorkin A (2003) Oligomerization of dopamine transporters visualized in living cells by fluorescence resonance energy transfer microscopy. J Biol Chem 278:28274-28283.

Sorkina T, Hoover BR, Zahniser NR, Sorkin A (2005) Constitutive and protein kinase $\mathrm{C}$-induced internalization of the dopamine transporter is mediated by a clathrin-dependent mechanism. Traffic 6:157-170.

Sorkina T, Miranda M, Dionne KR, Hoover BR, Zahniser NR, Sorkin A (2006) RNA interference screen reveals an essential role of Nedd4-2 in dopamine transporter ubiquitination and endocytosis. J Neurosci 26:8195-8205.

Torres GE, Carneiro A, Seamans K, Fiorentini C, Sweeney A, Yao WD, Caron MG (2003) Oligomerization and trafficking of the human dopamine transporter. Mutational analysis identifies critical domains important for the functional expression of the transporter. J Biol Chem 278:2731-2739.

Wei Y, Williams JM, Dipace C, Sung U, Javitch JA, Galli A, Saunders C (2007) Dopamine transporter activity mediates amphetamine-induced inhibition of Akt through a Ca2+/calmodulin-dependent kinase IIdependent mechanism. Mol Pharmacol 71:835-842.

Wood W, Martin P (2002) Structures in focus-filopodia. Int J Biochem Cell Biol 34:726-730.

Yamashita A, Singh SK, Kawate T, Jin Y, Gouaux E (2005) Crystal structure of a bacterial homologue of $\mathrm{Na}(+) / \mathrm{Cl}(-)$-dependent neurotransmitter transporters. Nature 437:215-223.

Yguerabide J, Schmidt JA, Yguerabide EE (1982) Lateral mobility in membranes as detected by fluorescence recovery after photobleaching. Biophys J 40:69-75.

Zahniser NR, Sorkin A (2004) Rapid regulation of the dopamine transporter: role in stimulant addiction? Neuropharmacology 47 Suppl 1:80-91. 